\title{
Neonatal hypoxia-ischemia in rat elicits a region-specific neurotrophic response in SVZ microglia
}

\author{
Urs Fisch $^{1,2^{*}}$ D , Catherine Brégère ${ }^{2}$, Florian Geier ${ }^{3,4}$, Laurie Chicha ${ }^{2}$ and Raphael Guzman ${ }^{2,5,6}$
}

\begin{abstract}
Background: Recent findings describe microglia as modulators of neurogenesis in the subventricular zone (SVZ). SVZ microglia in the adult rat are thought to adopt a neurotrophic phenotype after ischemic stroke. Early postnatal microglia are endogenously activated and may therefore exhibit an increased sensitivity to neonatal hypoxiaischemia (HI). The goal of this study was to investigate the impact of cortico-striatal HI on the microglial phenotype, function, and gene expression in the early postnatal SVZ.

Methods: Postnatal day (P)7 rats underwent sham or right-hemispheric HI surgery. Microglia in the SVZ, the uninjured cortex, and corpus callosum were immunohistochemically analyzed at P10, P20, and P40. The transcriptome of microdissected SVZ and cortical microglia was analyzed at P10 and P20, and the effect of P10 SVZ microglia on neurosphere generation in vitro was studied.

Results: The microglial response to HI was region-specific. In the SVZ, a microglial accumulation, prolonged activation and phagocytosis was noted that was not observed in the cortex and corpus callosum. The transcriptome of SVZ microglia and cortical microglia were distinct, and after HI, SVZ microglia concurrently upregulated pro- and anti-inflammatory as well as neurotrophic genes. In vitro, microglia isolated from the SVZ supported neurosphere generation in a concentration-dependent manner.

Conclusions: Microglia are an inherent cellular component of the early postnatal SVZ and undergo developmental changes that are affected on many aspects by neonatal HI injury. Our results demonstrate that early postnatal SVZ microglia are sensitive to $\mathrm{HI}$ injury and display a long-lasting region-specific response including neurotrophic features.
\end{abstract}

Keywords: Microglia, Subventricular zone, Hypoxia-ischemia, Development, Neurogenesis, Rat

\section{Background}

Hypoxia-ischemic encephalopathy (HIE) is a neonatal brain injury most commonly caused by birth asphyxia. HIE occurs in 1-3 per 1000 live full-term births, but rises up to 40 per 1000 in preterm born with very low birth weight $[1,2]$. Of affected infants, $25 \%$ develop persistent neurological impairments, including cerebral palsy, disorders of cognition and behavior, sensation, motricity, and epilepsy [3]. Currently, hypothermia is the

\footnotetext{
* Correspondence: urs.fisch@unibas.ch

'Department of Neurology, University Hospital Basel, University Basel, Basel, Switzerland

${ }^{2}$ Brain ischemia and regeneration, Department of Biomedicine, University Hospital Basel, University Basel, Basel, Switzerland

Full list of author information is available at the end of the article
}

standard of care in patients with moderate to severe HIE, and different therapeutic approaches are investigated in clinical trials $[4,5]$.

The rodent model of neonatal hypoxic-ischemic encephalopathy (HI) reflects the key pathomechanisms of HIE in term-born infants and has been shown to be a strong inducer of early postnatal SVZ neurogenesis [68]. Up to now, there are no clinical therapies available that specifically modulate this induced neurogenesis to amplify repair processes. Therefore, further research on $\mathrm{HI}$-induced SVZ neurogenesis is needed.

Accumulating evidence indicates that microglial cells in the neurogenic niches bear an exclusive function as modulators of neural stem cell (NSC) and neural progenitor cell

(C) The Author(s). 2020 Open Access This article is distributed under the terms of the Creative Commons Attribution 4.0 International License (http://creativecommons.org/licenses/by/4.0/), which permits unrestricted use, distribution, and 
(NPC) proliferation and differentiation [9]. In the adult rat, SVZ microglia adopt a proneurogenic phenotype after ischemic stroke [10] and are therefore a potential therapeutic target to enhance regeneration after neonatal HI.

Microglia are the resident tissue macrophage of the central nervous system (CNS) and are essential to maintain homeostasis in health and to initiate immune responses in disease. Rodent microglia colonize the developing brain as early as E8.5 [11], before the CNS undergoes extensive developmental maturation. They play an active role in the CNS development by phagocytosis [12], synaptic pruning [13], regulation of axonal outgrowth, and positioning of interneurons [14] as well as trophic support of cortical neurons [15] and oligodendrocytes [16, 17]. Lately, the different stages of microglial development have been extensively characterized using genome-wide expression profiling. Up to eight different clusters of microglia appear to exist throughout development from E14.5 until late age, with the highest diversity being observed during embryonic and early postnatal development, when microglia are still differentiating [17-20].

Microglial diversity exists not only temporally during development, but also spatially in different brain regions [21], underscoring the necessity to study microglia in the context of their microenvironment. The knowledge about the effects of microglial sub-populations in the developing postnatal brain is still scarce. Within the first postnatal weeks, while "maturing", SVZ microglia are highly activated, proliferative, and phagocytic; display an amoeboid shape; and express the lysosomal marker CD68 [16, 22]. In contrast, in the physiological adult brain, microglia do not express CD68 anymore and are thought to have a more surveilling role of their environment [23].

HI brain injury may cause diverse reactions by distinct, yet immature microglial populations in the developing early postnatal brain. We hypothesized that after HI, SVZ microglia adopt a specific phenotype which may in some aspects be supportive for HI-induced SVZ neurogenesis, similar to the adult SVZ microglia after ischemic stroke. Therefore, this study provides an in-depth characterization of the microglial phenotype in the early postnatal rat SVZ and the impact of neonatal $\mathrm{HI}$ on their development.

\section{Methods}

\section{Hypoxia-ischemia neonatal rat model}

All animal experiments were approved by the local veterinarian authorities and complied with the Swiss animal welfare guidelines. Sprague Dawley rats were bred inhouse, and the day of birth was considered as P0. P7 rat neonates were randomly assigned to sham or HI surgery, with a balanced representation of both sexes among the two surgery groups. One hour prior to surgery, animals were injected intraperitoneally with buprenorphine (0.03 $\mathrm{mg} / \mathrm{kg}$ body weight). Anesthesia was induced with $5 \%$ isoflurane and maintained with 3\% during the surgery. The HI surgery consisted of a modification of the RiceVannucci model: the right common carotid artery was exposed and temporarily clipped with a Sugita 4-mm aneurysm mini clip (Mizuho) and the skin incision temporarily sutured. Animals recovered for $10 \mathrm{~min}$ on a heating pad and were then placed for $40 \mathrm{~min}$ into a hypoxic chamber constantly flushed with $8 \%$ oxygen/92\% nitrogen gas mixture $(2 \mathrm{~L} / \mathrm{min})$ that was immersed in a temperature-controlled water bath to maintain an air temperature of $37.5{ }^{\circ} \mathrm{C}$. During hypoxia, epileptic seizures were observed in some animals as previously reported [24] but were not an exclusion criterion for further analyses. Animals then recovered for $10 \mathrm{~min}$ on a heating pad and were briefly anesthetized with isoflurane for clip removal and permanent skin suture. A typical neonatal HI injury pattern consists of an ipsilateral cortico-striatal injury sparing out the medial regions of the hemisphere (Fig. 2a) and a chronic ventriculomegaly due to brain volume loss (Fig. 1b). The sham surgery consisted of artery exposure without temporary occlusion and without hypoxia exposure. A subgroup of sham-assigned animals underwent a "hypoxia only" procedure with sham surgery followed by hypoxia exposure as described above without temporary carotid artery clipping.

\section{BrdU administration and brain collection for stainings}

Animals received daily single intraperitoneal bromodeoxyuridine (BrdU) injections $(100 \mathrm{mg} / \mathrm{kg}$ body weight, Sigma) for three consecutive days after surgery and before sacrifice (Fig. 1a). Animals were then sacrificed at P10, P20, or P40 to reflect acute, subacute, and chronic stages after $\mathrm{HI}(n=5$ sham and $n=5 \mathrm{HI}$ per time point, three animals at P10 for "hypoxia only"). Transcardiac perfusion with $0.9 \%$ saline under deep anesthesia, followed by $4 \%$ paraformaldehyde (PFA) in $0.1 \mathrm{M}$ phosphate buffer $\mathrm{pH} 7.4(\mathrm{~PB})$ was performed. Brains were post-fixed in $4 \% \mathrm{PFA}$ in $\mathrm{PB}$ for $48 \mathrm{~h}$ at $4{ }^{\circ} \mathrm{C}$, cryoprotected in consecutive $15 \%$ and $30 \%$ sucrose solutions, embedded in OCT (Leica Biosystems), and cryosectioned. Coronal free-floating sections $(30 \mu \mathrm{m})$ were stored at $-20{ }^{\circ} \mathrm{C}$ in a cryoprotectant solution (30\% ethylene glycol, $30 \%$ sucrose in $\mathrm{PB}$ ) until staining.

\section{Cresyl violet staining and animal selection for histological studies}

Coronal brain sections (interval $180 \mu \mathrm{m}$ ) were mounted on slides (Superfrost plus, Menzel), stained with 0.1\% cresyl violet acetate (Sigma), and scanned (Nikon Eclipse TI-E microscope). Brain sections including the anterior 

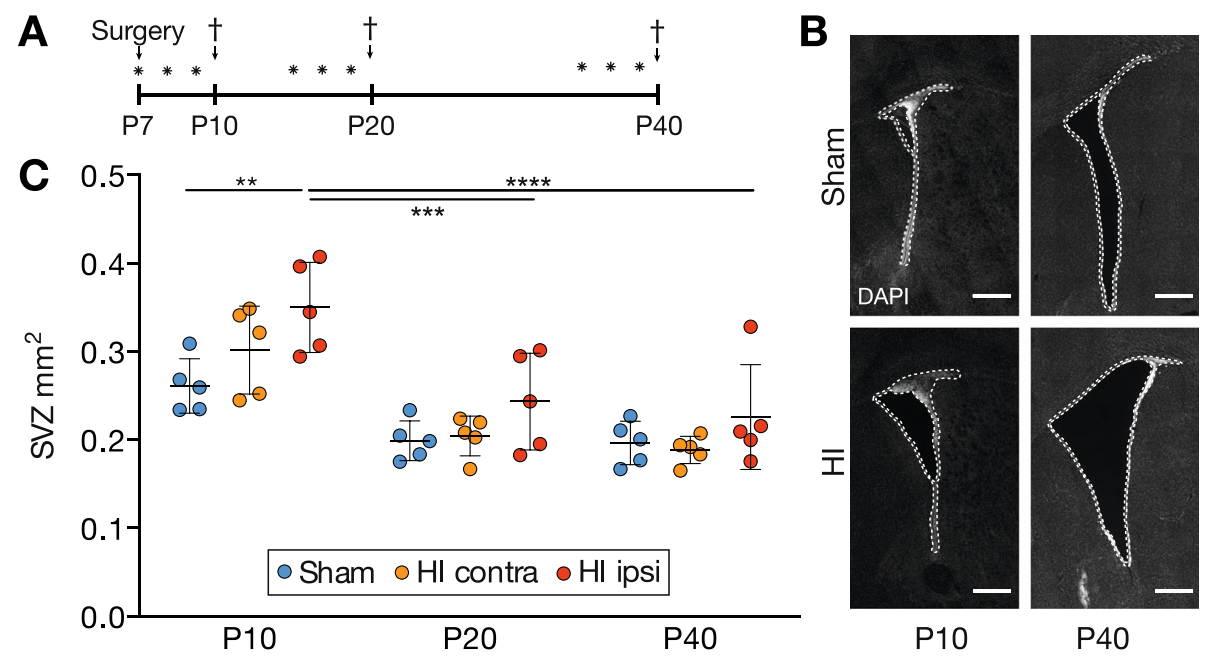

Fig. 1 The SVZ temporarily expanded in size after neonatal $\mathrm{HI}$. a Experimental timeline for the in vivo studies. Animals were subjected to sham or $\mathrm{HI}$ surgery at P7 and sacrificed at P10, P20, or P40. BrdU was injected for three consecutive days before sacrifice (asterisk). b Representative images of the ipsilateral SVZ after sham and HI surgery at P10 and P40. c Quantification of the SVZ size after sham and HI surgery. Individual data shown as dots, bars as mean with SD (error bar). Two-way ANOVA with Tukey post hoc test, ${ }^{* *} p<0.01,{ }^{* * *} p<0.001,{ }^{* * * *} p<0.0001$ (Additional file 1: Table S3). Scale bar for b, $500 \mu \mathrm{m}$

SVZ and 0.40 rostral and -0.20 caudal to bregma in P10 rats [25] (corresponding anatomical sections for P20 and P40 rats, respectively) were investigated. Due to the significant variability in $\mathrm{HI}$ injury size in the Rice-Vannucci model of neonatal HIE, two investigators (UF, CB) independently assessed the HI injury size using ImageJ software (version 2.00rc.43/1.50e) and their results were averaged. The size of HI injury was calculated by subtracting the intact area in the right, from here on defined as the ipsilateral hemisphere from the total area of the left, contralateral hemisphere in 3 serial cresyl violet-stained sections as previously described [6]. Animals with no apparent or extensive $\mathrm{HI}$ injury that affected the SVZ were excluded in order to compare relatively homogenous groups. Thus, per time point, $n=5$ sham animals and $n=$ $5 \mathrm{HI}$ with mild to moderate injury severity were selected (total of 30 with 17 females, 7 excluded), and $n=3$ (2 females) P10 "hypoxia only" (Additional file 1: Table S1).

\section{Immunostaining}

For immunohistochemistry, brain sections were washed in Tris-buffered saline (TBS), incubated in blocking buffer (TBS with $2 \%$ fish gelatine, $0.3 \%$ Triton X-100, Sigma) for $1 \mathrm{~h}$ at room temperature (RT), then incubated with primary antibodies (Table 1) overnight at $4{ }^{\circ} \mathrm{C}$ in blocking buffer, washed repetitively with TBS, and incubated with the corresponding species-specific Alexa-Fluor $(488,546,555,647)$-conjugated donkey or goat $(\mathrm{H}+\mathrm{L})$ secondary antibodies (Thermo Fisher) in blocking buffer (1:2000) for $1 \mathrm{~h}$ at RT. Sections were counterstained with $0.5 \mu \mathrm{g} / \mathrm{ml}$ DAPI (Sigma) before mounting on superfrost slides with ProLong Gold (Thermo Fisher). For BrdU labeling, sections were pretreated with $2 \mathrm{~N} \mathrm{HCl}$ for $30 \mathrm{~min}$ at RT before the blocking step. For proliferating nuclear cell antigen (PCNA) labeling, antigen retrieval was performed for $5 \mathrm{~min}$ at $80{ }^{\circ} \mathrm{C}$ in target retrieval solution (Dako) before blocking.

Table 1 Primary antibodies used for stainings

\begin{tabular}{llll}
\hline Host/primary antibody & Producer & Concentration for histology & Concentration for cytology \\
\hline Mouse/anti-BrdU & BD Bioscience & $1: 200$ & $1: 2000$ \\
Mouse/anti-CD68 & R\&D Systems & $1: 2000$ & $1: 1000$ \\
Guinea pig/anti-DCX & Merck Millipore & $1: 2000$ & $1: 1000$ \\
Rabbit/anti-lba1 & Wako & $1: 1000$ & $1: 500$ \\
Goat/anti-Iba1 & Abcam & & \\
Rabbit/anti-Ki67 & Abcam & $1: 1000$ & \\
Mouse/anti-Nestin & Millipore & $1: 700$ & \\
Rabbit/anti-PAX6 & Biolegend & & \\
Mouse/anti-PCNA & DAKO & &
\end{tabular}


For immunocytochemistry (Table 1), cell culture wells were fixed for $15 \mathrm{~min}$ in $4 \%$ PFA at RT. The immunofluorescence steps were identical to those described above.

\section{Brain section image acquisition and analysis}

The 20x wide-field immunofluorescence images from brain sections were acquired on an Eclipse TI-E microscope (Nikon), or 40x confocal immunofluorescence image stacks $(330 \times 330 \mu \mathrm{m}, 11 \mu \mathrm{m}$ depths, step size $0.5 \mu \mathrm{m})$ on a CSU-W1 microscope (Visitron Systems). Representative microglial activation images were acquired on a 40x LSM 710 confocal microscope (Zeiss). All quantitative analyses were conducted in three brain sections (interval $180 \mu \mathrm{m}$ ), and mean values were calculated per animal. Image acquisition settings were identical for all stainings.

\section{SVZ area and microglial quantification}

Quantifications of SVZ area and microglial morphology, density, and activation were performed manually with ImageJ from wide-field images. The SVZ area was defined by its DAPI+ cellular density (Fig. 1b). The microglial cell morphology was classified into three categories: (i) amoeboid with roundish shape without processes, (ii) intermediate with irregular shape and short processes, and (iii) ramified with processes extending more than twofold the diameter of the cell body (Fig. 4 A1-A3). The microglial density was calculated as the number of ionized calcium-binding adapter molecule 1 (Iba1) + cells per area in three brain regions: (i) the SVZ, (ii) a $1600 \times$ $200 \mu \mathrm{m}$ rectangle including layers I-VI of the M2 supplementary motor cortex (CX), and (iii) in the sum of three $150-\mu \mathrm{m}$ squares which were symmetrically distributed in the midline corpus callosum (CC) (Fig. 2a). Microglial activation, here defined by expression of CD68 [16], was calculated as the number of CD68+ Iba1+ cells per total number of Iba1+ cells for each region. The analysis was conducted in five animals per surgery and time point.

\section{Quantification of cell proliferation and microglial ball-and- chain buds}

Paired box 6 protein (PAX6)+ PCNA+ cells in the most medial part of the dorsolateral SVZ were manually counted in a confocal microscopy stack of $50 \mu \mathrm{m} \times 50 \mu \mathrm{m}$ $\times 10 \mu \mathrm{m}$ depth using ImageJ. Only cell nuclei being fully included within the stack were counted. Microglial proliferation (number of BrdU+ Iba1+ or Ki67+ Iba1+ cells per total number of Iba1+ cells) and ball-and-chain buds (number of ball-and-chain-shaped pouches per total number of Iba1+ cells) in the dorsolateral SVZ or CX were manually quantified in 3D-reconstructed confocal stack images with Imaris software (ver. 7.6.5, Bitplane). Ball- and-chain buds were defined as spherically shaped extensions at the terminal branch of microglial processes. Analysis was conducted in three animals per surgery and time point.

\section{Microglial isolation and RNA purification}

Animals underwent sham or HI surgery at P7, and microglia were isolated from the SVZ and CX at P10 or P20 for subsequent transcriptome analysis $(n=6$ animals per surgery and time point, total of 24 with 11 females; 38 excluded [Additional file 1: Table S1]). Animals were deeply anesthetized and perfused with ice-cold $0.9 \%$ saline. Brains were extracted and kept in ice-cold Hank's balanced salt solution (HBSS) (Sigma) for the following sterile procedures. The region of the anterior SVZ was coronally cut with razor blades in 2-mm-thick sections and two sections selected. Sections from HI animals were further processed only if the $\mathrm{HI}$ injury severity was mild to moderate without affection of the ipsilateral SVZ or the adjacent medial striatum and corpus callosum (microscopic assessment during preparation). From individual $\mathrm{HI}$ animals, the ipsidorsolateral SVZ and the contralateral CX were microdissected under a dissection microscope (Leica) and separately collected. From individual sham animals, SVZ or CX from both hemispheres were microdissected. The individual tissue samples were processed at $4{ }^{\circ} \mathrm{C}$. They were washed (centrifugation at $300 \mathrm{~g}$ for $5 \mathrm{~min}$, followed by aspiration of the supernatant), dissociated with the papain neural dissociation kit (Miltenyi), filtered through a 40- $\mu \mathrm{m}$ strainer, washed again, and magnetically labeled with mouse anti-rat CD11b microbeads (1:200) (Miltenyi) for $20 \mathrm{~min}$. CD11b+ cells were ferromagnetically isolated using MS columns (Miltenyi) following the manufacturer's instructions. The purity of CD11b+ sorted cells was tested by flow cytometry (see below). Due to the very small tissue samples and consecutively low cell yield, simultaneous flow cytometry analysis and RNA collection from individual tissue samples were not possible. Isolated CD11b+ cells were immediately processed with the Arcturus PicoPure RNA isolation kit (Thermo Fisher Scientific) including DNase treatment according to the manufacturer's instructions and stored at $-80^{\circ} \mathrm{C}$ after extraction buffer treatment. RNA isolation was then completed batch-wise. RNA integrity and concentration from CD11+ isolated cells were measured with the RNA 6000 Pico Kit (Agilent) on the 2100 Bioanalyzer (Agilent).

\section{Microglial transcriptome analysis}

Sample preparation for microarray processing was performed externally at Life \& Brain $\mathrm{GmbH}$, Bonn, Germany. In brief, $500 \mathrm{pg}$ total RNA per sample was reverse transcribed into cDNA using the GeneChip WT Pico Assay Kit (Affymetrix) in a two-step process according to the manufacturer's instructions. The cDNA was subsequently 


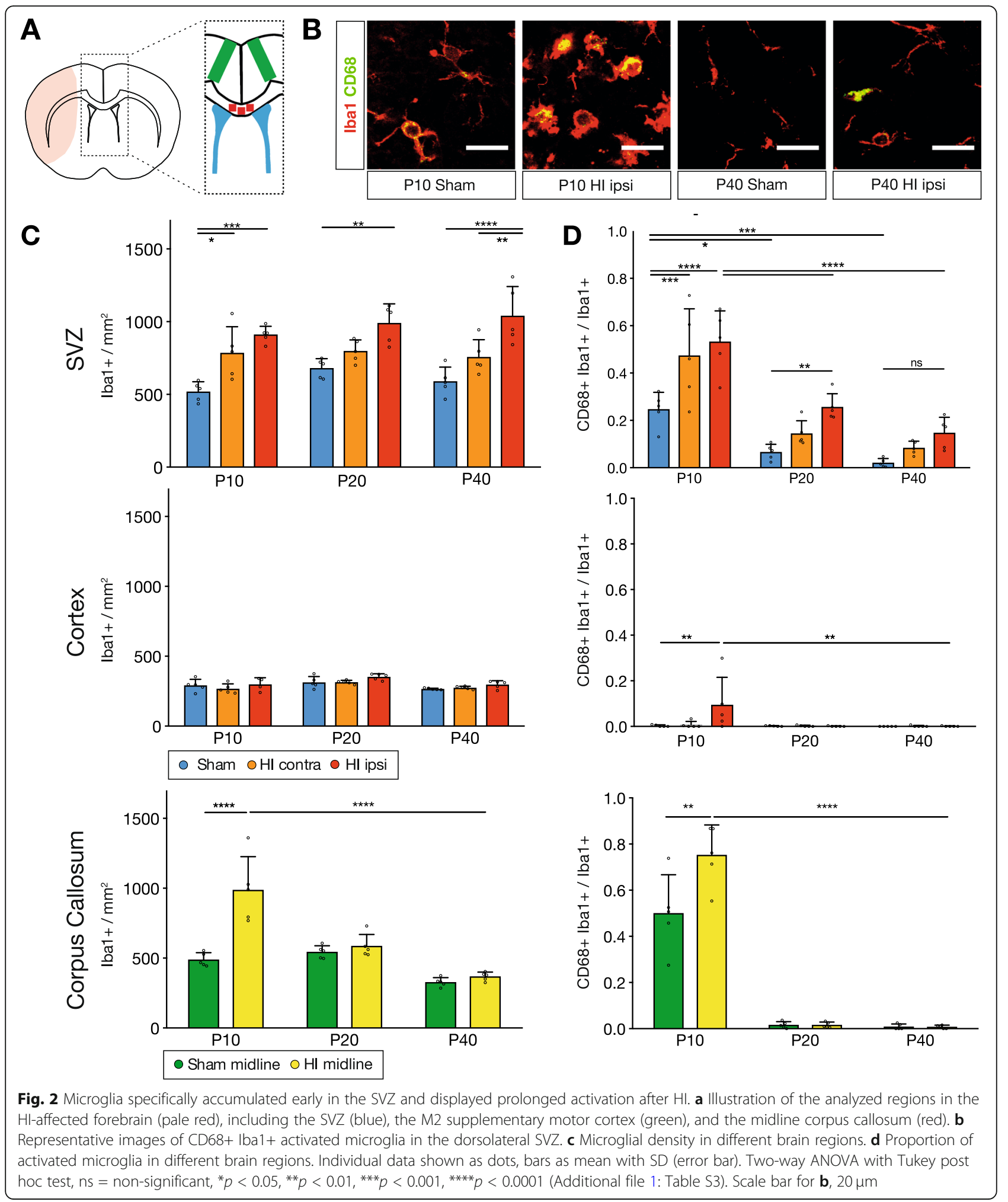

fragmented, labeled, and hybridized to the GeneChip Rat Transcriptome Array 1.0 (Affymetrix). After staining, scanning was performed on a GeneChip Scanner 3000 (Affymetrix).
The raw microarray data was normalized using the R/ Bioconductor package oligo (version 1.38.0). In brief, CEL files were read-in and subsequently normalized using the function rma. Probe sets were annotated with 
the affycoretools package (version 1.46.4). Entrez IDs with multiple probe sets were filtered for the probe set with the highest variance. One sample was identified as an outlier, and the animal (P20 sham SVZ) was removed from further analysis. The limma package (version 3.30.7) was used for differential gene expression analysis. One set of contrasts was defined to evaluate the surgery differences at both time points. $P$ values were adjusted for multiple testing using the Benjamini and Hochberg false discovery rate (FDR), and genes with a FDR $<0.05$ were considered as significant. Published microarray data sets were used to define M1 and M2 polarization markers by selecting the 15 most differentially expressed genes (FDR < 0.05 ) of lipopolysaccharide (LPS) or IL-4 stimulated microglia [26]. Gene sets defined in the Kyoto Encyclopedia of Genes and Genomes (KEGG) (version downloaded on 9 February 2017) were tested for differential enrichment using the limma function kegga. Principal component analysis and pathway-enrichment graphs were generated using $\mathrm{R}$ base graphics while heat maps for specified gene sets were based on the ComplexHeatmap package (version 1.12.0). Gene enrichment analysis for Gene Ontology (GO) terms for differentially expressed gene sets of SVZ and CX microglia were performed with the Database for Annotation, Visualization and Integrated Discovery (DAVID) v6.7.

\section{Quantitative real-time PCR}

The microarray results were validated by quantitative real time-polymerase chain reaction ( $\mathrm{qPCR}$ ) from pooled RNA samples from isolated SVZ microglia as described above that were not included in the microarray analysis (500 pg per sample, $n=2$ animals for sham, $n=2$ for HI per time point, respectively [Additional file 1 : Table S1]). Due to the low RNA amount (1 ng) per pooled sample group, we chose Igf-1 expression levels for validation. Pooled RNA was reverse-transcribed with the QuantiTect Reverse Transcription Kit (Qiagen), and qPCR was performed in triplicates using Fast SYBR Green MasterMix (Roche) on a LightCycler 480 (Roche). Primers for Igf- 1 and $\beta$-actin were commercially acquired (QuantiTect, Qiagen). All samples were analyzed concurrently in one experiment. Runs were normalized to the housekeeping gene $\beta$-actin by measuring $\Delta \mathrm{CT}$. The $2^{-\Delta \Delta C T}$ method was used for the calculation of the Igf- 1 fold change (FC) of the HI vs. sham group per time point.

\section{Primary neurosphere generation from SVZ tissue and microglial depletion}

Animals underwent sham or HI surgery at P7 (3 independent experiments with $n=3$ sham animals and $n=6$ HI per experiment, total of 27 with 15 females; 4 excluded [Additional file 1: Table S1]) and were deeply anesthetized and decapitated at P10. Rat heads were immersed in 70\% ethanol for $30 \mathrm{~s}$ and kept in ice-cold sterile HBSS until sterile dissection. Coronal sections of $6 \mathrm{~mm}$ thickness of the anterior SVZ were prepared as described above. Sections from $\mathrm{HI}$ animals were further processed only if the $\mathrm{HI}$ injury severity was mild to moderate without affection of the ipsilateral SVZ or the adjacent medial striatum and corpus callosum (microscopic assessment during preparation). Rectangular tissue blocks including the whole ipsior contralateral SVZ and the adjacent medial striatum were isolated. From HI animals, ipsi- and contralateral tissue blocks were pooled separately, whereas from sham animals, all SVZ tissue blocks were pooled per experiment, resulting in three tissue groups: (i) ipsilateral HI, (ii) contralateral HI, and (iii) sham (Fig. 6 A1). The pooled tissue samples were washed, dissociated with the papain neuronal dissociation kit (Miltenyi), and filtered through a $70-\mu \mathrm{m}$ strainer. The cell suspension was washed and counted (TC20 counter, Bio-Rad). Dissociated cells were seeded at a density of 0.3 Mio in uncoated 24-well plates (Corning). Cells were incubated in $500 \mu \mathrm{l}$ neuronal expansion medium (DMEM/F12 1:1, Gibco; with N2 supplements [human apo-Transferrin, $100 \mathrm{mg} / \mathrm{l}$; insulin, $25 \mathrm{mg} / \mathrm{l}$; putrescine, $100 \mu \mathrm{M}$; sodium selenite, $30 \mathrm{nM}$; Sigma]; penicillin/streptomycin, $100 \mu \mathrm{g} / \mathrm{ml}$, Gibco; glutaMAX, $2 \mathrm{mM}$, Gibco). The cell culture plates were incubated at $37^{\circ} \mathrm{C}$ in $5 \% \mathrm{CO}_{2}$. The medium was completely replaced after $2 \mathrm{~h}$ and plates treated in three different conditions: (i) addition of microglia-targeting mouse anti-CD11b saporinconjugated antibodies (anti-CD11b SAP, $0.35 \mu \mathrm{g} / \mathrm{ml}$ ) (Advanced Targeting Systems), (ii) unspecific mouse IgG saporin-conjugated antibodies (IgG SAP, $0.35 \mu \mathrm{g} / \mathrm{ml}$ ) (Advanced Targeting Systems), or (iii) no addition of antibodies (control) (Fig. 6 A2). Cell cultures were supplemented daily with recombinant human fibroblast growth factor $(20 \mathrm{ng} / \mathrm{ml}, \mathrm{R} \& D$ Systems $)$ and recombinant human epidermal growth factor $(20 \mathrm{ng} / \mathrm{ml}$, Peprotech). Half of the medium was replaced at 3 days in culture (DIC). Neurospheres were analyzed after 6 DIC.

\section{Cell culture image acquisition and analysis}

Two $2.5 \mathrm{x}$ wide-field images per well were acquired on an Axiovert 200 microscope (Zeiss), representing 25\% of the total well surface. Number and area of individual neurospheres with a diameter $>45 \mu \mathrm{m}$ were automatically counted using ImageJ. For each experimental condition, the data from three wells were averaged and extrapolated to calculate neurosphere numbers per well. Qualitative confocal 40x fluorescence images were acquired from immunocytochemically stained wells.

\section{Flow cytometry}

Dissociated samples were washed and stained with mouse anti-CD11b-FITC (1:200) (Bio-Rad) and mouse 
anti-CD45-PE (1:200) (Bio-Rad) in buffer solution (phosphate-buffered saline $\mathrm{pH} 7.4,0.5 \%$ bovine serum albumin, $2 \mathrm{mM}$ ethylenediaminetetraacetic acid [EDTA]) at $4{ }^{\circ} \mathrm{C}$ for $20 \mathrm{~min}$, followed by washing and processed on an Accuri flow cytometer (BD Bioscience). All data were analyzed with FlowJo software (version 10.2).

\section{Statistical analysis}

All data sets except the microarray data were analyzed in Prism software (GraphPad, version 6). All data are presented as mean \pm standard deviation (SD) if not stated otherwise. Statistical analysis was performed with two-way ANOVA with Tukey post hoc test for age or surgery, or one-way ANOVA with Holm-Sidak post hoc test. $P$ values $<0.05$ were considered as significant. Correlations were computed with two-tailed Pearson correlation coefficients or nonparametric Spearman correlation. Countings for the SVZ size, microglial density, and proportion of activated microglia from ipsi- and contralateral sham hemispheres did not show any significant differences (Additional file 1: Figure S1). Thus, only ipsilateral (right-sided) sham data were used for statistical analysis of histological data.

\section{Results}

\section{The SVZ transiently enlarges after $\mathrm{HI}$}

Neonatal HI was shown to induce a temporary enlargement of the SVZ and an increase in NSC/NPC proliferation $[27,28]$. We measured the area of the total SVZ in sham and $\mathrm{HI}$ animals at P10, P20, or P40, reflecting acute, subacute, and chronic stages of HI injury (Fig. 1a, b). Due to the significant variability in HI injury severity in the Rice-Vannucci model of neonatal HIE, HI injury size was independently assessed by two investigators (Pearson coefficient $r=0.96,95 \%$ confidence interval 0.91-0.98, $p<0.0001, n=34$ ) and animals selected with mild to moderate HI injury severity (median $49 \%$ hemispheric injury size [interquartile range 35-60\%]) without histological signs of HI damage to the anterior SVZ.

$\mathrm{HI}$ induced an enlargement of the SVZ at P10 that was most prominent in the ipsilateral HI SVZ as compared to the sham SVZ $(0.35$ [mean] \pm 0.05 [SD] vs. 0.26 $\pm 0.03 \mathrm{~mm}^{2}, p<0.004$ ) (Fig. 1c). This enlargement was transient, and at P2O and P40, the HI ipsilateral SVZ normalized again to the size of sham SVZ (for P40, 0.23 \pm 0.06 vs. $0.20 \pm 0.02 \mathrm{~mm}^{2}$ ), whereas ventriculomegaly persisted (Fig. 1b). Overall, the size of the SVZ markedly decreased with age with an early transient enlargement in the ipsilateral hemisphere of $\mathrm{HI}$ animals.

\section{Microglia in the SVZ specifically accumulate early and remain activated after $\mathrm{HI}$}

After ischemic stroke in the adult rat, microglia in the ipsilateral SVZ become activated and accumulate over weeks [10]. Early postnatal microglia are not yet fully mature and are endogenously activated in the SVZ [1620, 22], a developmental feature which may affect their response to injury. We therefore analyzed the impact of $\mathrm{HI}$ on microglia in the SVZ, CC, and CX by quantifying their density and the proportion of activated microglia (Fig. 2a, b).

After HI, microglia in the ipsilateral SVZ significantly accumulated at P10 (ipsilateral HI SVZ, $911 \pm 57$ vs. sham SVZ, $519 \pm 67 \mathrm{Iba} 1+/ \mathrm{mm} 2, p<0.0001)$. This early increase in microglial density in HI SVZ remained constant until P40 when compared to sham SVZ (ipsilateral HI SVZ $1041 \pm 200$ vs. sham SVZ $590 \pm 66, p<0.0001$ ) (Fig. 2c). Likewise, HI led to a striking increase in the proportion of activated microglia in the ipsilateral SVZ at P10 (ipsilateral HI SVZ $0.53 \pm 0.13$ vs. sham SVZ 0.25 $\pm 0.07 \mathrm{CD} 68+\mathrm{Iba} 1+/ \mathrm{Iba} 1+, p<0.0001)$ and P20 (ipsilateral HI SVZ $0.26 \pm 0.06$ vs. sham SVZ $0.06 \pm 0.03, p<$ 0.006 ), while overall, the proportion of activated microglia decreased in both groups with age (Fig. 2d). However, with increasing age, a substantial reduction of activated microglia was observed in both $\mathrm{HI}$ ipsilateral SVZ (P10, $0.53 \pm 0.13$ vs. P40, $0.08 \pm 0.03, p<0.0001$ ) and sham SVZ (P10, $0.25 \pm 0.07$ vs. P40, $0.02 \pm 0.02, p \leq$ 0.001 ). Thus, microglia in the ipsilateral SVZ responded to $\mathrm{HI}$ with early accumulation and prolonged activation, while during the same period, the number of PCNA+ PAX6+ NPCs remained unchanged (Additional file 1: Figure S2).

We next investigated whether these findings were specific to the SVZ and quantified the same parameters in the adjacent $C X$ and CC (Fig. 2a). In the CX, the microglial density of HI vs. sham remained unchanged from P10 (ipsilateral HI CX, $299 \pm 47$ vs. sham CX, $310 \pm 49$ Iba1 $+/ \mathrm{mm}^{2}$ ) until P40 (ipsilateral HI CX, $297 \pm 28$ vs. sham $C X, 266 \pm 6)$, while in the CC, it transiently increased at P10 (HI CC, $989 \pm 238$ vs. sham CC, $490 \pm$ $49, p<0.0001)$ but thereafter returned to sham levels until P40 (HI CC, $367 \pm 31$ vs. sham CC, $328 \pm 32$ ) (Fig. 2c). At P10, HI led to an increased proportion of activated microglia in the $\mathrm{CX}$ (ipsilateral $\mathrm{HI} C X, 0.1 \pm 0.12$ vs. sham $\mathrm{CX}, 0.003 \pm 0.004 \mathrm{CD} 68+\mathrm{Iba} 1+/ \mathrm{Iba} 1+, p<$ $0.003)$ and in the CC (HI CC, $0.75 \pm 0.13$ vs. sham CC, $0.5 \pm 0.17, p<0.01$ ); nevertheless, by P20, the proportion of activated microglia was equally reduced in all groups (Fig. 2d).

In summary, microglia in regions other than the SVZ were activated for a shorter time as compared to SVZ microglia and did not demonstrate a sustained accumulation. Interestingly, the SVZ contralateral to the ischemic hemisphere showed similar results as the ipsilateral HI SVZ, although less pronounced (Figs. 1c and 2c, d). To verify that the findings in the contralateral SVZ were due to the combination of global hypoxia and unilateral ischemia and not to hypoxia alone, rat neonates were 
subjected to sham surgery followed by exposure to hypoxia ( $n=3$ "hypoxia only" animals). The results in this group were similar to those measured in sham animals, underscoring the specificity of the microglial response to the HI injury in the ipsilateral and contralateral SVZ (Additional file 1: Figure S3).

\section{SVZ microglia proliferate early after $\mathrm{HI}$}

We then asked if HI-induced microglial accumulation in the SVZ was due to local proliferation. BrdU was administered for three consecutive days before sacrifice, and proliferating BrdU+ microglia were quantified in the dorsolateral SVZ (Fig. 3a). The proportion of proliferating microglia in sham animals was higher at P10 than afterwards. HI led to a significant increase in microglial proliferation at P10 in the ipsilateral and contralateral SVZ (ipsilateral HI, $0.16 \pm 0.04$ vs. sham, $0.11 \pm 0.01$ BrdU+ Iba1+/Iba1+, $p<0.014$ ) (Fig. 3b). The analysis of Ki67+ Iba1+ proliferating cells at P10 depicted similar, but non-significant trends (Additional file 1: Figure S4), suggesting that the HI-induced proliferation mainly occurred before P10. In comparison, microglial proliferation in the CX was noticeably lower and remained unaffected by $\mathrm{HI}$ at P10 (ipsilateral HI, $0.06 \pm 0.03$ vs. sham, $0.05 \pm 0.01)$ and was practically inexistent thereafter (data not shown). Hence, microglia proliferated early after birth and more pronounced in the SVZ after HI.

HI temporarily increases the proportion of amoeboid SVZ microglia

Microglia in the dorsolateral SVZ were classified based on their morphology into amoeboid, intermediate, and ramified phenotypes (Fig. 4 A1-A3). An amoeboid morphology is associated with increased activation, proliferation, and phagocytosis [23]. In both sham and HI animals, the proportion of amoeboid microglia decreased with age (Fig. 4B). At P10, HI caused a striking increase in the percentage of amoeboid microglia in both ipsilateral and contralateral SVZ (ipsilateral HI, $68.9 \pm 25.2 \%$; contralateral HI, $49.2 \pm 27.6 \%$; sham, $6.8 \pm 7.4 \%, p \leq$ $0.01)$. However, this difference was no longer present afterwards (P40, ipsilateral HI, $0.9 \pm 0.3 \%$; contralateral HI, $1.4 \pm 1.6 \%$; sham, $2.6 \pm 1.1 \%$ ). In opposite, the proportion of ramified microglia was significantly lower at P10 after HI compared to sham (ipsilateral HI, $14.5 \pm$ 16.5\%; contralateral HI, $14.3 \pm 11.0 \%$; sham, $69.4 \pm 9.1 \%$, $p \leq 0.001$ ), but no longer at P40 (ipsilateral HI, $87.9 \pm$ 0.6\%; contralateral HI, $84.6 \pm 12.4 \%$; sham, $93.4 \pm 2.3 \%$ ). In comparison, $\mathrm{CX}$ microglia in $\mathrm{HI}$ and sham animals remained ramified from P10 until 40 (data not shown). Thus, the specific endogenous activation of microglia in the early postnatal SVZ was markedly amplified by HI.

\section{Increased number of ball-and-chain buds among SVZ microglia after HI}

Phagocytosis is a key feature of microglia in the developing brain and maintains homeostasis in the adult neurogenic niches mediated through ball-and-chain-shaped pouches that engulf and phagocyte pyknotic cells [29, 30]. We therefore assessed microglial ball-and-chain buds in the dorsolateral SVZ which are best detectable in ramified microglia (Fig. 4C, D1-D5, E1-E4) [29].

In the sham SVZ, the number of ball-and-chain buds among Iba1+ cells were most prominent at P10 and continuously decreased thereafter (P10, $0.52 \pm 0.1$ vs. P40, $0.01 \pm 0.1, p \leq 0.0002$ ) (Fig. 4F). This developmental course was reversed in $\mathrm{HI}$ animals (ipsilateral HI SVZ; $\mathrm{P} 10,0.06 \pm 0.06$ vs. $\mathrm{P} 40,0.38 \pm 0.15, p<0.003)$. Thus, at $\mathrm{P} 40$, microglia in the SVZ from HI animals showed significantly more ball-and-chain buds than in that from sham animals (ipsilateral SVZ, $0.38 \pm 0.15$ vs. sham SVZ,

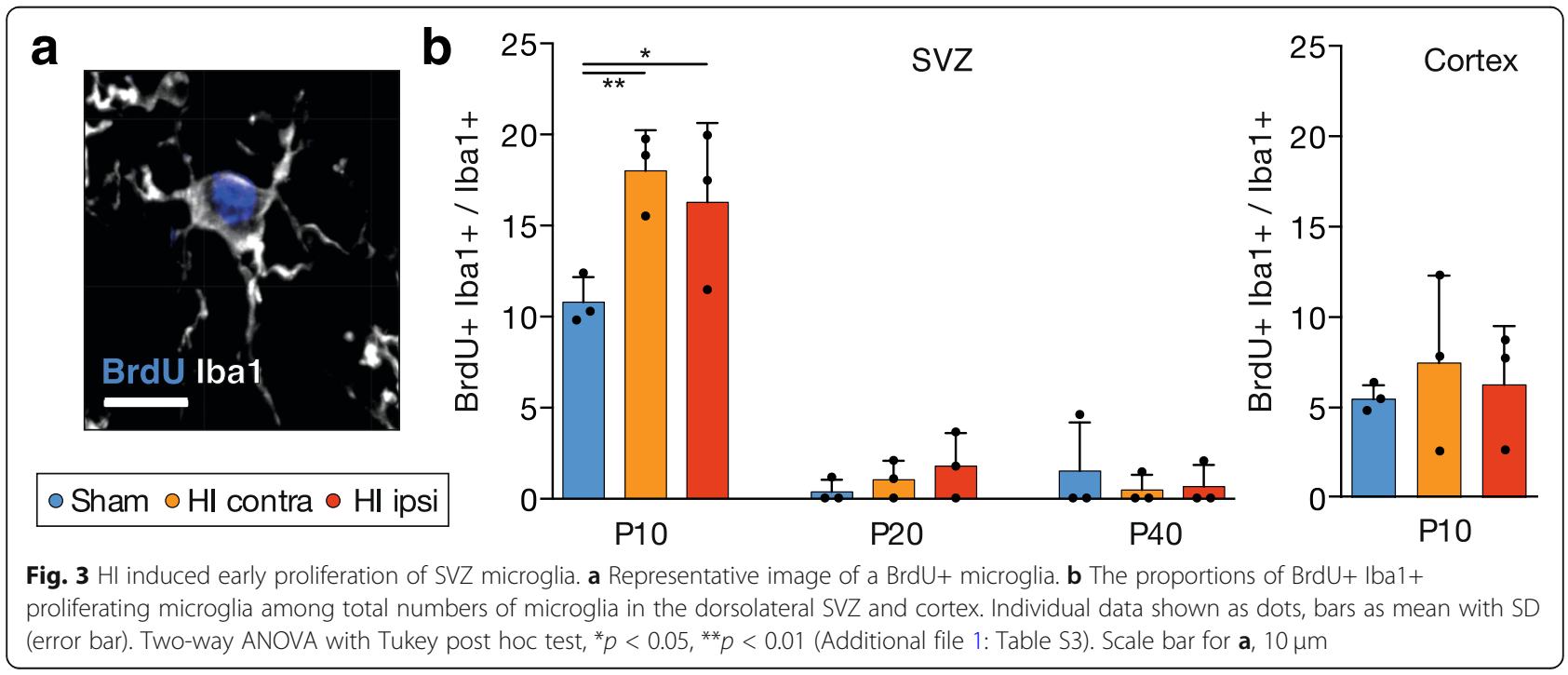



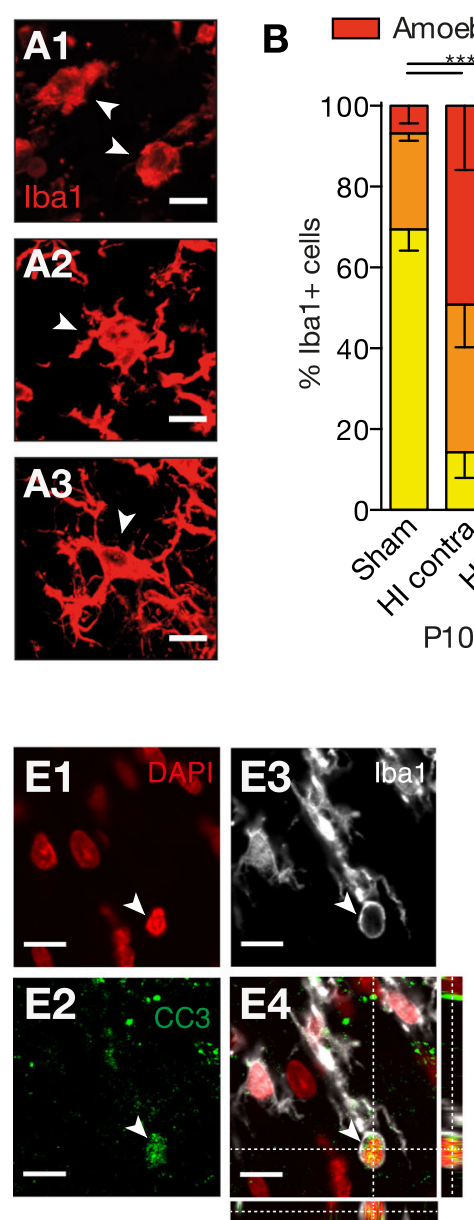

B

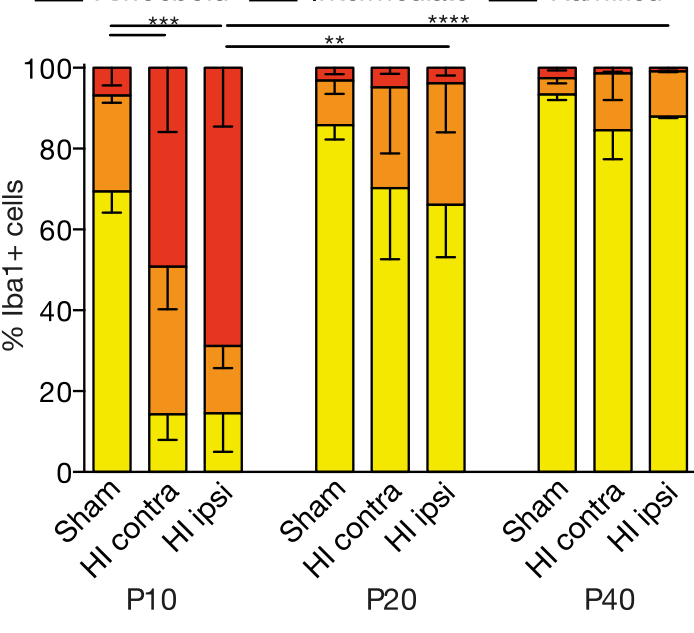

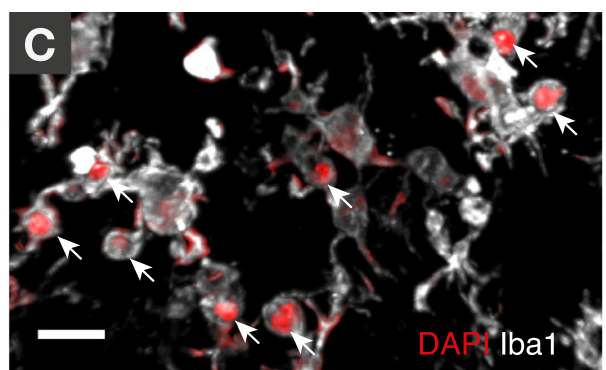
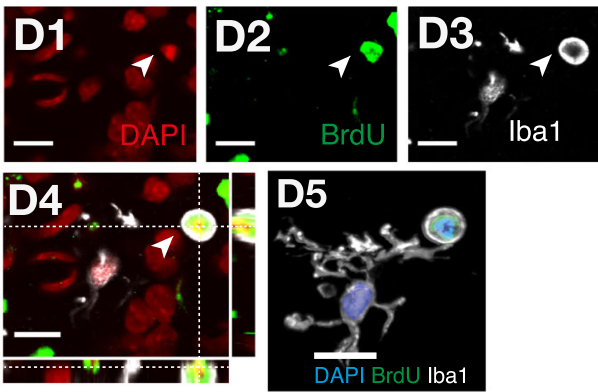

$\mathbf{F}$

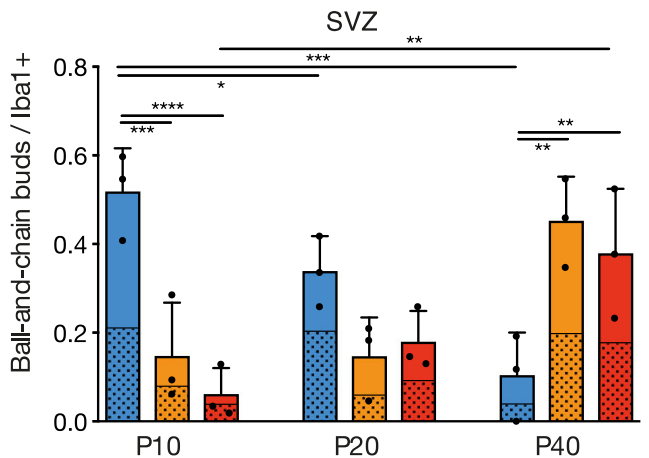

Cortex

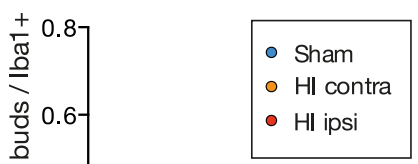

Fig. $4 \mathrm{HI}$ affects microglial morphology and phagocytosis. A1, A2, A3 Microglia were classified upon their morphology to A1 amoeboid, A2 intermediate, or $\mathbf{A} \mathbf{3}$ ramified (arrows). B Proportions of Iba1+ microglia in the dorsolateral SVZ according to their morphology. Statistical analysis for ramified microglia. Mean with standard error of the mean (error bar). C 3D-reconstructed representative image highlighting the content of ball-and-chain microglial buds (arrows) that consist of pyknotic DAPI+ cells. D1, D2, D3, D4, D5 Representative images of a pyknotic BrdU+ cell engulfed by a microglial bud (arrow) with D1 DAPI, D2 BrdU, D3 Iba1 stainings, D4 the correspondent composite stack image, and D5 a 3Dreconstructed image of the whole microglia cell. E1, E2, E3, E4 Representative images of a pyknotic CC3+ cell engulfed by a microglial bud (arrow) with E1 DAPI, E2 CC3, E3 Iba1 stainings, and E4 the correspondent composite stack image. F Number of ball-and-chain buds per Iba1+ microglia in the dorsolateral SVZ and the cortex. The polka dot-filled bar area indicates the mean number of BrdU+ containing ball-and-chain buds. Individual data shown as dots, bars as mean with SD (error bar). Two-way ANOVA with Tukey post hoc test, ${ }^{*} p<0.05,{ }^{* *} p<0.01$, ${ }^{* * *} p<$ $0.001,{ }^{* * * *} p<0.001$ (Additional file 1: Table S3). Scale bar for A, C, D, E, $10 \mu \mathrm{m}$

$0.01 \pm 0.1, p<0.009)$. Approximately half of the balland-chain buds contained BrdU+ nuclei (Fig. 4 D1-D5, F), indicating that the engulfed cells had recently proliferated. Microglial ball-and-chain buds in the CX were rare at P10 (Fig. 4F) and undetectable afterwards (data not shown). Engulfed cells rarely stained for the apoptotic marker cleaved caspase 3 (CC3) (Fig. 4E1-E4). They remained negative for NSC and NPC markers (sex determining region Y-box 2 [SOX2], doublecortin [DCX], PAX6, polysialylated-neural cell adhesion molecule [PSA-NCAM], data not shown) which may be due to rapid protein degradation [29], or alternatively other cells were engulfed. In conclusion, among ramified microglia, ball-and-chain buds were most prominent early at P10 in sham animals and late at P40 in HI animals.

SVZ microglia upregulate pro- and anti-inflammatory and neurotrophic genes after $\mathrm{HI}$

We analyzed the transcriptome of $\mathrm{Cd} 11 \mathrm{~b}+$ cells microdissected and purified from the SVZ or CX of individual sham and HI animals at P10 and P20. Flow cytometry analysis demonstrated a concentration of $>95 \% \mathrm{CD} 11 \mathrm{~b}+$ CD45+ cells after isolation (Additional file 1: Figure S5). Total RNA was extracted from these cells (RIN $\geq 7.2$ [mean 9.6]), and a total of $500 \mathrm{pg}$ RNA per sample was 
used for microarray experiments. The microarray analysis revealed high expression of microglia-specific transcripts, including C1qa, Cx3cr1, P2ry12, and Tmem119 among all samples, whereas the macrophage-specific gene expression was low (Fig. 5a). The principal component analysis demonstrated a grouping of most samples of the same condition with age and anatomical origin being the main components before surgery (Fig. 5b). The microarray results were validated by qPCR of additional SVZ samples that were not used in the microarray (Additional file 1: Figure S6).

The difference in gene expression of SVZ and CX microglia was age-dependent. In P10 sham animals, microglia in the SVZ significantly $($ FDR $<0.05)$ upregulated 2319 and downregulated 1362 genes compared to microglia in the CX. In contrast, at P20, they were only 151 significantly upregulated and 33 downregulated genes. Some upregulated genes in SVZ microglia were consistent with increasing age, including Axl, Clec7a, Fabp7, Lrrc74b, Olr1, Olr1366, Rab7b, Sulf2, and Zcchc18, while other gene upregulations were only found at a specific time point, such as at Cd8b, Dcx, Gpnmb, Gpr165, Hsdl2, Igf1, Ms4a6bl, Pdk4, and Spp1 at P10.

We next performed gene-annotation enrichment analysis for significantly and minimally 1.5 -fold upregulated genes in SVZ microglia in comparison with CX microglia (Fig. 5c). At P10, the resulting GO terms in sham microglia were associated with cell motility, nervous system development, and axonal guidance, in difference to metabolic and inflammatory processes and negative regulation of apoptosis in $\mathrm{HI}$ animals. At P20, in sham microglia, only the GO term myelination was present, whereas in $\mathrm{HI}$ microglia, the GO terms associated with cell motility, nervous system development, and negative regulation of neuronal regulation shared some resemblance to those found in sham microglia at P10.

Thus, the difference of the SVZ and CX microglial gene expression underscored the dynamics and regionspecificity of microglial development. The geneannotation enrichment analysis indicated a long-term impact of HI onto SVZ microglial gene expression and potential functional effects.

We then analyzed in further detail gene expression differences in the SVZ. HI microglia significantly upregulated 1807 and downregulated 389 genes compared to sham at P10, and upregulated 2642 and 7288 downregulated genes at P20, respectively. Overall, the FC of significantly differentially expressed genes in the SVZ was modest. In HI compared to sham microglia, 19 genes were more than 2 -fold upregulated and 3 downregulated at P10, and 25 upregulated and none downregulated at P20. The 25 most upregulated genes are listed in Table 2 for P10, and Table 3 for P20, respectively. Among those genes, we noticed a concurrent expression of genes associated with proinflammatory (RasGef1b, TNFsf9, Rab32, Stat1, Ifi27, Pdpn) and antiinflammatory (Anxa2, CD206, Gpnmb, Clec7, miR-146a) effects as well as genes with neurotrophic effects such as Igf-1 [15] and C3 [31]. Thus, the transcriptome of SVZ microglia after HI shows a complex pattern of concurrent upregulation of pro- and anti-inflammatory as well as neurotrophic genes.

\section{SVZ microglia do not polarize to a M1/M2 state after HI but enrich pathways of neurodegenerative diseases}

We examined whether after HI, SVZ microglia express markers associated with the M1 or M2 polarization state. An analysis based on mined microarray data [26] did not support a clear distinction at any given time point (Fig. 5d). We then performed a KEGG pathway analysis of differentially expressed genes of $\mathrm{HI}$ and sham SVZ microglia (Fig. 5e). This analysis revealed in HI SVZ microglia an enrichment of pathways associated with neurodegenerative diseases, including oxidative phosphorylation, ribosome, Alzheimer's (AD), Parkinson's, and Huntington's disease. Interestingly, these pathways were also enriched in microglial transcriptome studies in murine models for AD [32] and amyotrophic lateral sclerosis (ALS) [33]. A comparative analysis had previously identified a consensus microglial transcriptional profile among different models of neurodegenerative diseases that was distinct from a LPS-stimulated inflammatory microglial response [32]. Multiple genes of this consensus profile, including Anxa2, Anxa5, Gpnmb, Mfsd1, Igf-1, Clec7, Rsad2, Eif2ak2, Stat1, and C3, were upregulated in SVZ microglia compared to CX microglia with emphasis on $\mathrm{HI}$ microglia at P10 (Additional file 1: Figure S7).

\section{Microglial depletion reduces neurosphere formation}

Neurospheres derived from the neonatal SVZ after HI grow in higher numbers than those from sham SVZ, reflecting the HI-induced neurogenesis [28]. By conditional depletion, we explored if microglia affect this HIinduced neurosphere formation. We focused on the P10 time point where the HI-mediated effects on SVZ microglia were most striking.

The SVZ from P10 HI or sham animals were microdissected and pooled in three tissue groups (Fig. 6 A1): (i) ipsilateral HI, (ii) contralateral HI, and (iii) sham. Cell cultures from each tissue group were subjected to three different conditions (Fig. 6A2): (a) control (no microglial depletion), (b) IgG (addition of a non-specific saporinconjugated antibody), or (c) anti-CD11b (addition of a CD11b-specific saporin-conjugated antibody). After 6 DIC, neurospheres were formed and expressed nestin and DCX (Fig. 6B). At this stage, CD11b+CD45+ microglia from all tissue groups were highly depleted in the 
a

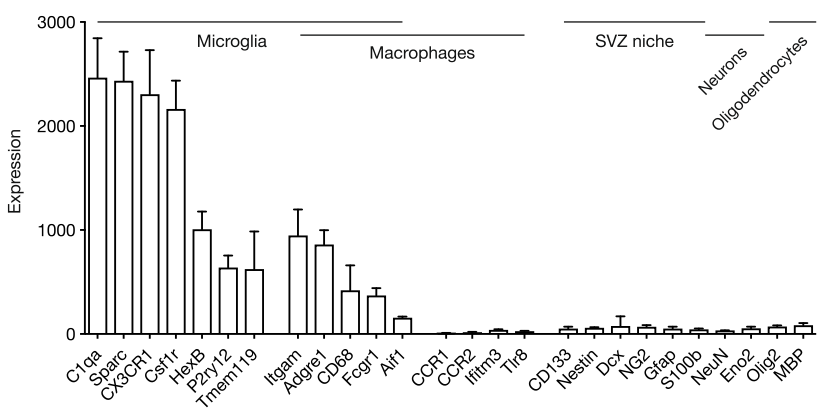

b

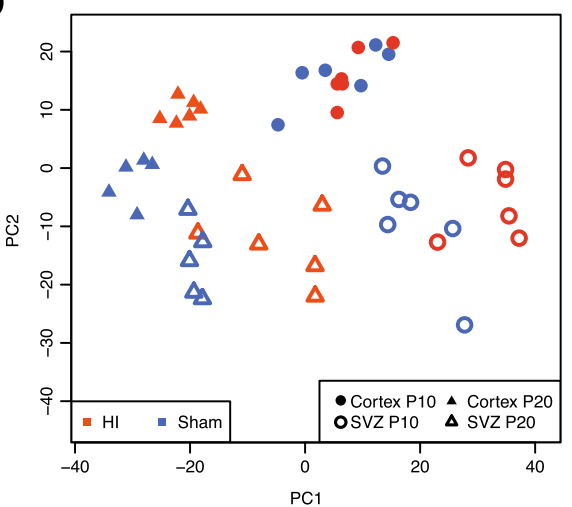

C

d

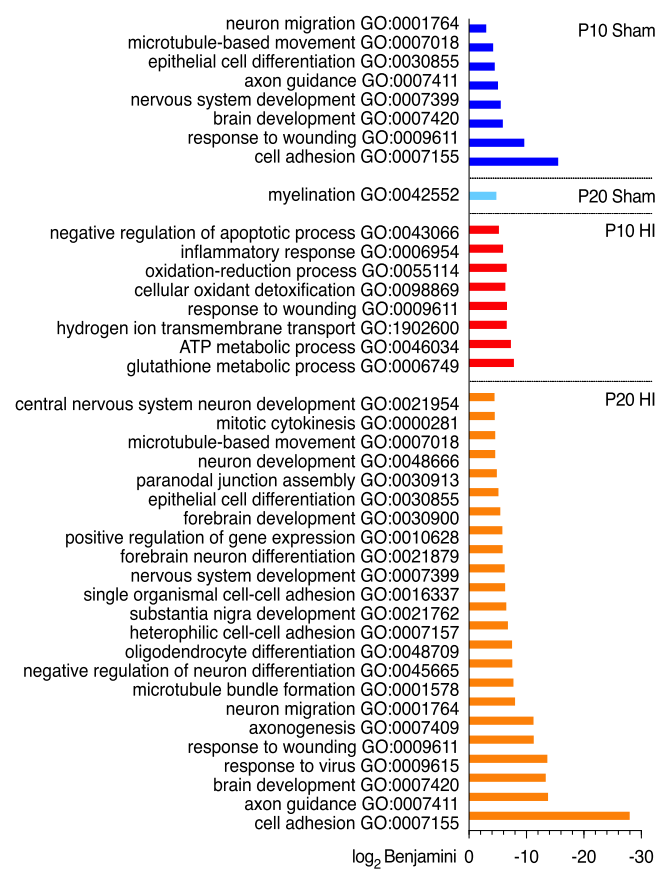

M1 Polarization (LPS)

M2 Polarization (IL-4)

P10

P20

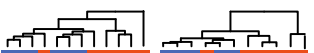

P10 P20
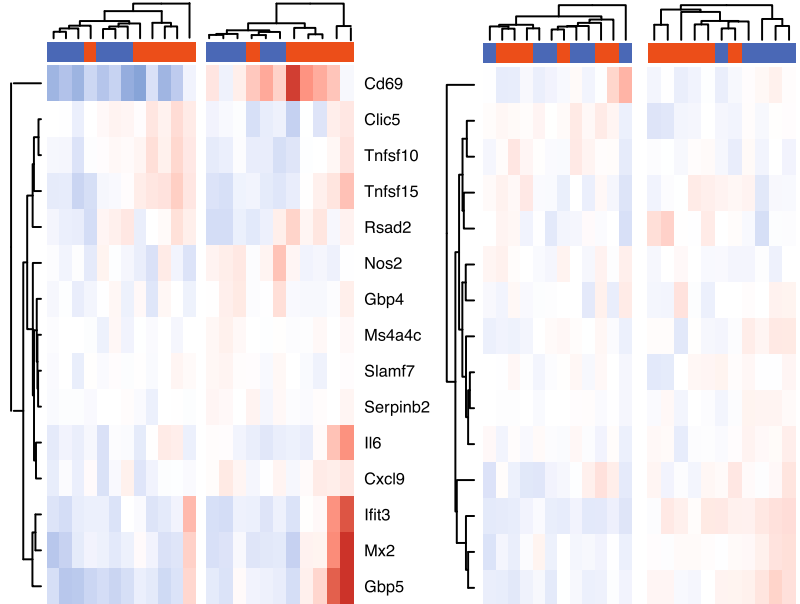

Rasgrp3

Gmnc

Gpr65

Cd300lb

Mtss1

Retnla

Cxcr2

Rab3il1

Rnase2

Sesn1

Cnr2

Cdh1

Chi313

Tmem26

Clec10a

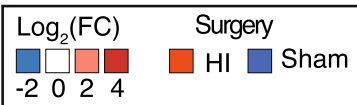

e

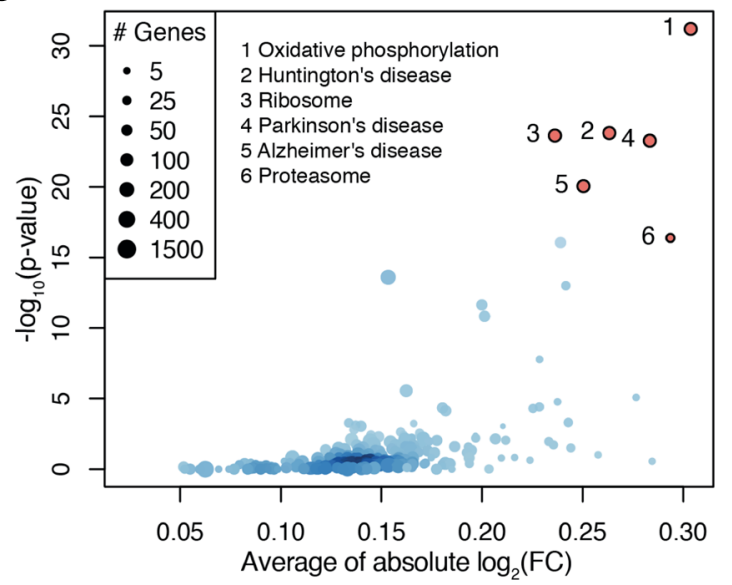

P20 HI vs. Sham

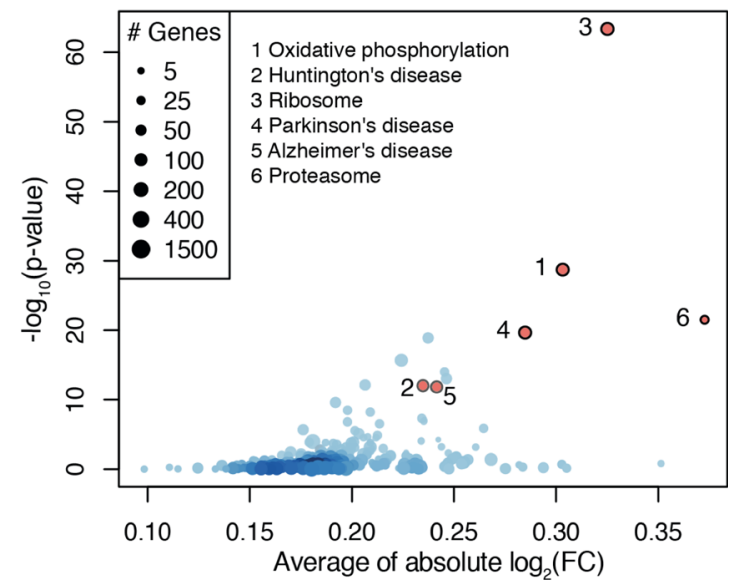

Fig. 5 (See legend on next page.) 
(See figure on previous page.)

Fig. 5 SVZ microglia upregulate pro- and anti-inflammatory and neurotrophic genes after $\mathrm{HI}$. a Expression of specific genes for different cell types in microdissected CD11 b+ microglia from the SVZ and cortex. Bars as mean with SD (error bar). b Principal component analysis of all samples. $\mathbf{c}$ Gene annotation enrichment analysis for significantly and > 1.5-fold upregulated genes in SVZ microglia compared to cortex microglia. $\mathbf{d}$ Heat maps for SVZ microglial expression of M1 (LPS)-polarized or M2 (IL4)-polarized genes. e KEGG pathways enrichment in SVZ microglia

anti-CD11b culture condition (mean 98\% reduction) when measured with a flow cytometer (Additional file 1: Table S2). Immunocytochemical stainings confirmed these results with microglia abundantly attached as single cells or part of neurospheres in the control and IgG conditions, and only few microglia detectable within the neurospheres in the anti-CD11b condition (Fig. 6C). Thus, microglia were an abundant part of the neurosphere culture and were effectively depleted by the antiCD11b antibody condition.

Next, we quantified the number of neurospheres (Fig. $6 \mathrm{D}, \mathrm{E})$. In the control condition, neurosphere numbers were increased in ipsilateral $\mathrm{HI}$ compared to sham tissue cultures (ipsilateral HI, $2308 \pm 363$ vs. sham, $1714 \pm 193$ neurospheres/well, $p<0.027)$. The IgG condition resulted in similar numbers as the control culture condition among all tissue groups, indicating that the non-specific saporinconjugated antibody did not impair neurosphere formation. Strikingly, treatment with the anti-CD11b antibody led to a significant decrease in neurosphere numbers in all tissue groups, when compared to control culture conditions $(p<0.027)$. Furthermore, we noted a positive correlation between $\mathrm{CD} 11 \mathrm{~b}+\mathrm{CD} 45+$ microglial cell fractions and neurosphere numbers (Spearman coefficient $r=0.87$, 95\% CI $0.68-0.93, p<0.0001, n=27$ ) among all experiments (Fig. 6F).

In summary, these findings indicated that CD11b+ CD45+ SVZ microglia supported neurosphere generation in vitro in a concentration-dependent manner. Cultures with ipsilateral SVZ microglia resulted in the highest

Table 2 Upregulated genes in SVZ microglia after HI at P10

\begin{tabular}{|c|c|c|c|}
\hline Gene symbol & Gene name & EntrezID & FC HI vs. sham \\
\hline$\overline{E m b}$ & Embigin & 114511 & 4.59 \\
\hline LOC100911403 & Membrane-spanning 4-domains subfamily A member 4A-like & 100911403 & 4.03 \\
\hline Ms4a6c & Membrane-spanning 4-domains, subfamily A, member $6 \mathrm{C}$ & 690930 & 2.76 \\
\hline Anxa2 & Annexin A2 & 56611 & 2.74 \\
\hline LOC290595 & Hypothetical gene supported by AF152002 & 290595 & 2.73 \\
\hline Ms4a4a & Membrane-spanning 4-domains, subfamily A, member 4A & 361734 & 2.61 \\
\hline CD206 & Mannose receptor, C type 1 & 291327 & 2.58 \\
\hline Lpl & Lipoprotein lipase & 24539 & 2.48 \\
\hline Ccl6 & Chemokine (C-C motif) ligand 6 & 287910 & 2.42 \\
\hline Anxa5 & Annexin A5 & 25673 & 2.28 \\
\hline Sdc4 & Syndecan 4 & 24771 & 2.24 \\
\hline Stom & Stomatin & 296655 & 2.19 \\
\hline Rasgef1b & RasGEF domain family, member 1B & 100361238 & 2.19 \\
\hline Cd8b & CD8b molecule & 24931 & 2.18 \\
\hline Gpnmb & Glycoprotein nmb & 113955 & 2.09 \\
\hline Tnfsf9 & Tumor necrosis factor superfamily member 9 & 353218 & 2.09 \\
\hline Atp6v1e1 & ATPase $\mathrm{H}+$ transporting $\mathrm{V} 1$ subunit E1 & 297566 & 2.06 \\
\hline Rab32 & RAB32, member RAS oncogene family & 365042 & 2.05 \\
\hline Mfsd1 & Major facilitator superfamily domain containing 1 & 361957 & 2.01 \\
\hline Slirp & SRA stem-loop interacting RNA binding protein & 688717 & 1.99 \\
\hline $\operatorname{lgf}-1$ & Insulin-like growth factor 1 & 24482 & 1.99 \\
\hline Pygl & Liver glycogen phosphorylase & 64035 & 1.98 \\
\hline $\operatorname{lgsf6}$ & Immunoglobulin superfamily, member 6 & 171064 & 1.98 \\
\hline Enpp6 & Ectonucleotide pyrophosphatase/phosphodiesterase6 & 306460 & 1.95 \\
\hline Tnfaip2 & TNF alpha induced protein 2 & 299339 & 1.95 \\
\hline
\end{tabular}

The 25 most upregulated genes in ipsilateral SVZ microglia after HI compared to sham SVZ microglia at P10 (FDR < 0.05) 
Table 3 Upregulated genes in SVZ microglia after HI at P2O

\begin{tabular}{|c|c|c|c|}
\hline Gene symbol & Gene name & EntrezID & FC HI vs. sham \\
\hline LOC100910270 & Uncharacterized & 100910270 & 6.37 \\
\hline Slfn4 & Schlafen 4 & 114247 & 4.51 \\
\hline Plac8 & Placenta-specific 8 & 360914 & 4.12 \\
\hline RT1-Da & RT1 class II, locus Da & 294269 & 3.06 \\
\hline RGD1563091 & Similar to OEF2 & 500011 & 2.59 \\
\hline Clec7a & C-type lectin domain family 7 , member $A$ & 502902 & 2.57 \\
\hline Rsad2 & Radical S-adenosyl methionine domain containing 2 & 65190 & 2.54 \\
\hline Clec12a & C-type lectin domain family 12, member A & 680338 & 2.51 \\
\hline Ifi27 & Interferon, alpha-inducible protein 27 & 170512 & 2.45 \\
\hline Tlr1 & Toll-like receptor 1 & 305354 & 2.26 \\
\hline Pdpn & Podoplanin & 54320 & 2.18 \\
\hline Eif2ak2 & Eukaryotic translation initiation factor 2-alpha kinase 2 & 54287 & 2.17 \\
\hline Stat1 & Signal transducer and activator of transcription 1 & 25124 & 2.17 \\
\hline LOC102553917 & Putative zinc finger protein 724-like & 102553917 & 2.16 \\
\hline Ifi27|2b & Interferon, alpha-inducible protein 27 like 2B & 299269 & 2.16 \\
\hline RT1-Ba & RT1 class II, locus Ba & 309621 & 2.14 \\
\hline F10 & Coagulation factor $X$ & 29243 & 2.14 \\
\hline ND3 & NADH dehydrogenase subunit 3 & 26199 & 2.12 \\
\hline LOC100911190 & Probable ATP-dependent RNA helicase DDX60-like & 100911190 & 2.11 \\
\hline Oas1b & 2-5 oligoadenylate synthetase $1 \mathrm{~B}$ & 246268 & 2.09 \\
\hline C3 & Complement C3 & 24232 & 2.07 \\
\hline LOC679827 & Similar to Ran-specific GTPase-activating protein & 679827 & 2.06 \\
\hline Mir146a & microRNA 146a & 100314241 & 2.06 \\
\hline Cxcl17 & C-X-C motif chemokine ligand 17 & 308436 & 2.05 \\
\hline$M \times 2$ & MX dynamin like GTPase 2 & 286918 & 2.02 \\
\hline
\end{tabular}

The 25 most upregulated genes in ipsilateral SVZ microglia after $\mathrm{HI}$ compared to sham SVZ microglia at P20 (FDR < 0.05 )

number of neurospheres while microglia depletion significantly reduced them.

\section{Discussion}

Microglia in the SVZ are increasingly recognized as modulators of neurogenesis [9] and undergo crucial developmental stages in the early postnatal rodent brain [16-20, 22]. In this study, we characterized the phenotype of microglia in the SVZ and adjacent brain regions during early development in rats and investigated the impact of a neonatal $\mathrm{HI}$ injury. As HI injury severity bears a considerable variability, only animals with mild to moderate injury were included in our analysis. This allowed to describe a relatively homogeneous experimental cohort. While exact calculation of $\mathrm{HI}$ injury size was available for the histological experiments, severity grading for the transcriptomic and cell culture experiments were based on microscopical assessment during dissection, thus reflecting a limitation for a direct comparison of $\mathrm{HI}$ injury severity among the different experiments. Our results point to a SVZ-specific response of microglia with a complex gene expression pattern revealing both immunomodulatory and potential neurotrophic effects on neurogenesis.

\section{Sustained early accumulation and prolonged activation of SVZ microglia after HI}

In comparison with adjacent brain regions, early postnatal SVZ microglia showed a region-specific early accumulation and prolonged activation after $\mathrm{HI}$. Furthermore, a corresponding accumulation of proliferating NPCs within the SVZ could not be observed, suggesting that the microglial features are cell type-specific. Similar findings had been previously reported in the adult rat after $2 \mathrm{~h}$ of middle cerebral artery occlusion (MCAO) [10], although with major differences. In particular, the SVZ microglial response lasted over 16 weeks in the adult rat and was limited to the ipsilateral SVZ. In comparison, the response in the neonate evolved mainly within 4 weeks and included the contralateral SVZ that was evoked by the combination of hypoxia and ipsilateral ischemic injury, but not by hypoxia alone. An 

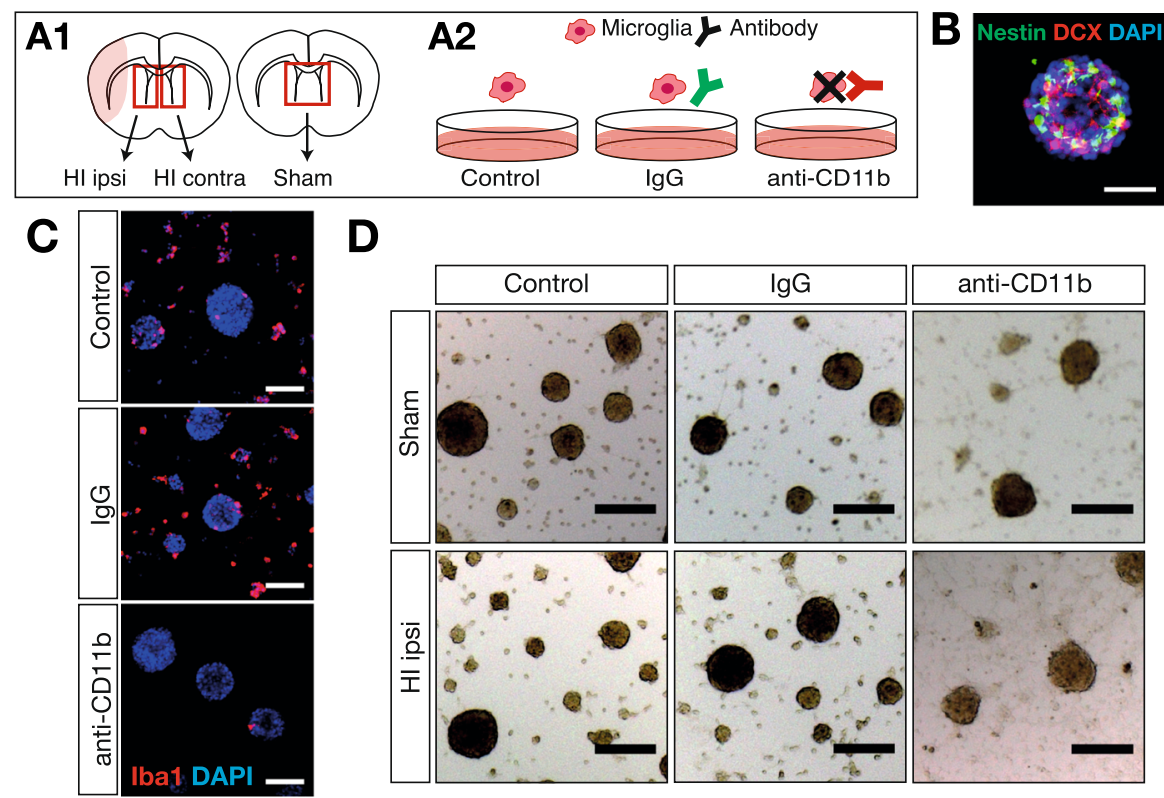

D

E

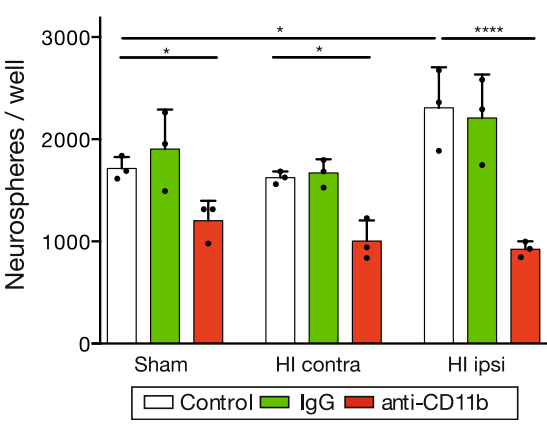

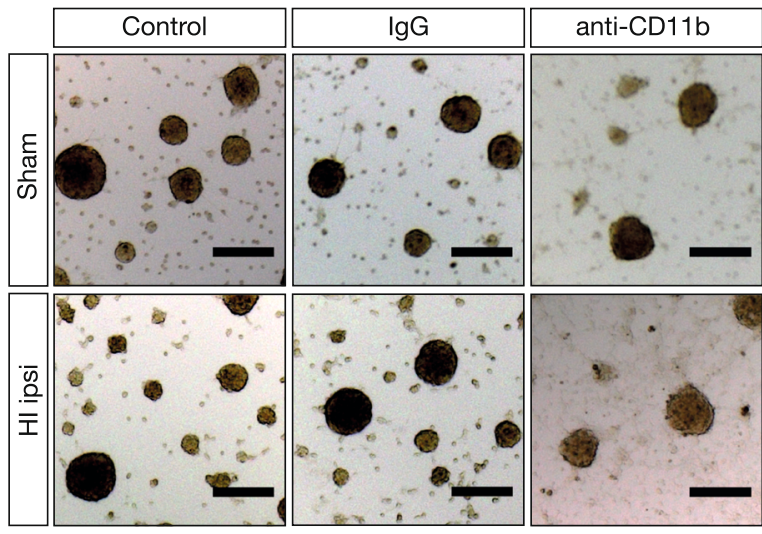

$\mathbf{F}$

$\mathbf{F}_{3000}$

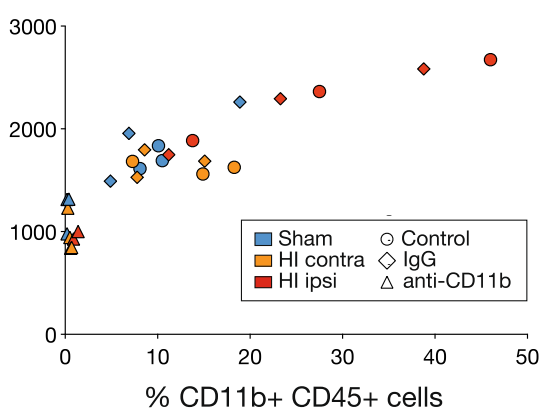

Fig. 6 Microglia depletion reduced neurosphere growth in vitro. A1, A2 Experimental design for a neurosphere assay with conditional depletion of microglia. A1 Tissue origin: ipsi- or contralateral tissue blocks including the SVZ were separately isolated from P10 HI animals (HI ipsi, HI contra), or pooled from P10 sham animals (sham). A2 Depletion method: dissociated tissue blocks were exposed to three conditions, with either no antibody (control), addition of nonspecific toxic antibodies (lgG), or CD11b+ specific toxic antibodies (anti-CD11b). B Representative image of a Nestin+ DCX+ neurosphere after 6 DIC. C Representative images of Iba1+ microglia in HI ipsi cell cultures after 6 DIC. The anti-CD11b condition strikingly reduced Iba1+ cells. D Representative images from sham and HI ipsi cell cultures after 6 DIC. Less neurospheres were present in the anti-CD11b conditions. E Number of neurospheres after 6 DIC. In the control and lgG conditions, HI ipsi-derived tissue cultures produced more neurospheres than sham-derived, reflecting $\mathrm{HI}$-induced proliferation and indicating no interference of unspecific toxic antibodies with cell growth. In contrast, anti-CD11b antibodies significantly decreased neurosphere numbers independent of tissue origin. $\mathbf{F}$ Data from $\mathbf{E}$ in comparison to microglial percentage at DIC6, showing a positive correlation between microglial proportions and neurospheres $(r=0.87,95 \% \mathrm{Cl}$ 0.68-0.93). Individual data shown as dots; bars as mean with SD (error bar). One-way ANOVA with Holm-Sidak post hoc test, ${ }^{*} p<0.05$, ${ }^{* * *} p<$ 0.0001 (Additional file 1: Table S3). Scale bar for B $50 \mu \mathrm{m}$, C $100 \mu \mathrm{m}, \mathbf{D} 200 \mu \mathrm{m}$

increased neurogenesis in contralateral SVZ has been noted previously after neonatal HI [6, 7]. Several potential factors may account for these differences including the injury model, sex distribution, rat strains, and the cell counting method, which in our study considered changes in SVZ size. Despite these and probably more factors, we propose that a major factor between the two studies is the developmental stage of the SVZ microglia which leads to a different time course of accumulation and activation of early postnatal and adult microglia. Notably, a study with 30 min MCAO was not able to reproduce the aforementioned findings in the adult rat
[34]. This suggests that stroke severity affects the adult SVZ microglial response. In our study, we found no correlation between the mild to moderate $\mathrm{HI}$ injury size and the SVZ microglial density and activation status (data not shown).

\section{Increased SVZ microglial proliferation and phagocytosis after $\mathrm{HI}$}

BrdU-labeling indicated an early endogenous SVZ microglial proliferation that is potentiated by HI injury. Whether this additional proliferation was solely responsible for the sustained early accumulation observed after 
HI or to which extent migrating myeloid cells contributed cannot be fully answered in this study. The gene expression analysis of CD11b+ SVZ cells showed high expression of signature microglial genes, thereby pointing out that the vast majority of these cells were of microglial origin.

In sham animals, the proportion of amoeboid microglia in the SVZ decreased continuously with age. HI injury temporarily increased this proportion in both the ipsilateral and contralateral SVZ before returning to levels similar to those in sham animals.

To our knowledge, microglial phagocytosis has not been quantified in the early postnatal SVZ. Microglial phagocytosis is fundamental for CNS development and maintenance, immune defense, and tissue repair. In mature ramified microglia, ball-and-chain-shaped phagocytic buds that engulf apoptotic cells from the proximity have been described [12, 29, 30, 35]. It is possible that ball-and-chain buds in ramified early postnatal microglia are also of the same phagocytic nature, although in contrast to the adult, they were not systematically demonstrating engulfement of cells that expressed markers for apoptosis or typical antigens for other SVZ cells. During physiological development, the proportion of ball-and-chain buds dramatically declined between P10 and P40, while it increased during the same period in HI-exposed animals. Of note, ball-andchain phagocytic terminal branches are best recognized in ramified microglia which are less present at earlier time points. Therefore, this analysis most likely underestimates the overall phagocytic activity at earlier time points, especially after HI, since non-ramified microglia are highly phagocytic [22]. It is speculated that the increased proportion of ball-and-chain buds after HI impacts the neurogenic niche, especially since a marked proportion of engulfed cells had recently proliferated. Even though the phagocytic nature of these microglial ball-and-chain protrusions is not fully ascertained, their increased proportion at P40 underlines the long-term effects of $\mathrm{HI}$ on SVZ microglia.

\section{Gene expression analysis indicates spatially and temporarily distinct patterns in SVZ microglia}

We performed a microarray transcriptome analysis of $\mathrm{CD} 11 \mathrm{~b}+$ microglia isolated from SVZ and cortex of individual sham and $\mathrm{HI}$ animals. Our results indicated a high purity of CD11b+ cells expressing microglial signature genes. In this analysis, neuroectodermal genes, and among them most prominently DCX, were also expressed by microglia, as previously reported $[17,36$, 37]. Whether this reflects a true microglial gene expression or the uptake of mRNA from neighboring cells remains to be established [37].

The microglial transcriptome showed significant differences between anatomical regions. Among the most upregulated genes in the SVZ were those recently described in pooled whole-brain transcriptome studies of early postnatal murine microglia $[17,19,20]$. Our data from individual rats add to these findings by identifying the SVZ microglia as a specific source for these gene patterns. However, the microarray data could not identify a CD11c+ subpopulation in the rat SVZ as previously reported in mice [17].

The gene enrichment analysis resulted in a clear difference between sham and HI SVZ microglia. Interestingly, categories concerning CNS development were present in sham animals at P10, and again, in HI animals at P20, suggesting common functional properties.

After HI, SVZ microglia upregulated both pro- and anti-inflammatory genes until P20. Importantly, microglial markers of acute inflammation such as IL-1 $\beta$, IL-6, TNF- $a$, and IFN- $\gamma[38-40]$ were not differentially expressed (data not shown), underscoring that the SVZ microglial response was different to that seen in microglia nearby ischemic tissue. Among the most upregulated genes in P10 SVZ microglia after HI were neurotrophic genes, such as IGF-1 and Gpnmb. Microglia-derived IGF-1 is essential for cortical neuronal survival [15], is neuroprotective after ischemic stroke [41], and is upregulated in the adult rat SVZ and striatal microglia after ischemic stroke [10, 34]. Gpnmb, also known as Osteoactivin, is predominantly expressed by microglia in the rodent brain $[42,43]$, especially in the early postnatal stage $[17,19]$. The role of Gpnmb in rat microglia is not fully understood. In mice, it shows antiinflammatory properties in macrophages [44] and neuroprotective effects in models of brain ischemia [45] and ALS [46]. In conclusion, these results point out to a spatially and temporally distinct gene expression pattern of SVZ microglia, which after $\mathrm{HI}$ is outlined by pro- and anti-inflammatory as well as neurotrophic genes.

\section{SVZ microglia display pathways associated with neurodegenerative diseases after $\mathrm{HI}$}

The M1 and M2 polarization state of microglia has recently been questioned by several in vivo microglial transcriptome studies [32, 33, 42, 47, 48]. Even though some characteristic polarization markers, such as CD206 or Stat1, were found among the most upregulated genes in HI-exposed SVZ microglia, the analysis did not indicate a clear trend to adopt a polarization state after neonatal HI.

The KEGG pathway enrichment analysis in SVZ microglia after HI pointed to pathways associated with neurodegenerative diseases. Interestingly, similar findings were reported in several microglia studies in mouse models for ALS, AD, accelerated, and physiological aging that were distinct from a microglial response to LPS exposure [32, 33, 49]. From these studies, a microglial 
consensus gene set, including Igf-1 and Gpnmb, was identified [32]. When applied to our study, this consensus gene set was mostly expressed in SVZ microglia at P10 with emphasis on HI-treated animals. This result was unexpected, and despite several methodological differences, it can be speculated that it may partially reflect a microglial state shared by radically distinct CNS pathologies $[48,50]$.

\section{SVZ microglia support neurosphere generation in vitro}

HI-induced proliferation of NSC and NPC can be recapitulated in an in vitro neurosphere assay. By specific depletion, we assessed the effect of SVZ microglia on neurosphere formation. In line with previous reports [28], cultures from ipsilateral HI SVZ display higher numbers of neurospheres than those from sham SVZ. Strikingly, this was completely abolished when microglia were depleted. Microglial percentage and neurosphere numbers in culture positively correlated among all experimental conditions, suggesting that microglia support neurosphere formation in a concentration-dependent manner.

These results are in line with previous in vitro studies demonstrating trophic effects of microglia. Conditioned medium from mixed cell cultures containing microglia from young and ischemic rodent brain tissue promoted NPC proliferation [51, 52]. In co-culture systems, microglia had inhibitory effects on NPC differentiation and attenuated negative effects of LPS or IFN- $\gamma$ on murine neurosphere formation [53, 54]. Also, secreted compounds stimulated neurosphere proliferation like IL-6, most likely derived from microglia [55], and IGF-1 [56], which we found to be specifically and highly expressed by SVZ microglia after HI. SVZ-derived NPCs establish functional gap junctions with microglia [57]. In our in vitro experiments, microglia were an integral part of neurospheres. Hence, it is possible that microglia modulate NPC not only via secreted factors but also through direct cell-cell interaction. Furthermore, the interaction between microglia and NPC is reciprocal with NPC enhancing microglial proliferation $[53,58]$, which is underlined by our results, where microglial proportions positively correlated with neurosphere numbers with the highest values in cultures from the ipsilateral HI SVZ. Future studies are warranted to identify the SVZ microglial mediators which enhance neurosphere formation after HI.

\section{Conclusions}

This report provides an in-depth characterization of the microglial phenotype in the rat SVZ after neonatal HI. In summary, microglia in the early postnatal SVZ are a distinct microglial sub-type in terms of morphology and gene expression profile. HI injury has lasting effects on their developmental course, as shown by their cellular accumulation, prolonged activation, and sustained phagocytosis, which do not occur in adjacent brain regions. After HI, SVZ microglia upregulate a heterogeneous pattern of genes related to pro- and anti-inflammatory as well as neurotrophic effects. This transcriptomic profile does not strictly reflect a M1 or M2 polarized state, but coincides to some extent with recently described microglial gene expression patterns found in several models of neurodegenerative diseases. Early postnatal microglia correlate with neurosphere generation in vitro in a concentration-dependent manner, suggesting a potentially reciprocal trophic effect.

HIE still bears a high risk for life-long sequelae for the affected patients. Thus, there is a need for therapies aiming at enhancing endogenous repair mechanisms. To evaluate such new therapeutic approaches, a better understanding of the cellular players in the pathomechanisms is necessary. Concurrent beneficial and detrimental effects mediated through microglia after CNS damage underscore the versatility, and thus the difficulty for their characterization. This study provides new insight into the specific response of early postnatal SVZ microglia upon HI injury. Our findings suggest that SVZ microglia might play a beneficial role for neurogenesis which merits further research. Overall, this study highlights the significance of the microenvironment and of the developmental stage on the microglial phenotype.

\section{Supplementary information}

Supplementary information accompanies this paper at https://doi.org/10. 1186/s12974-020-1706-y.

\footnotetext{
Additional file 1: Table S1. Total numbers of animals, numbers of included animals with sex distribution and excluded animals with reason for exclusion for each experiment. Table S2. Percentages of CD11b+ CD45+ microglia per tissue group and condition for each experiment. Table S3. List of all statistical comparisons in the main figures. Figure S1. Right and left hemispheres in sham animals are not different. Figure S2. Density of PCNA+ and PAX6+ cells in the dorsolateral SVZ. Figure S3. Hypoxia alone is insufficient to elicit HI-specific microglial changes. Figure S4. SVZ microglial phagocytosis of Ki67+ SVZ cells. Figure S5. Magnetic bead sorting resulted in a high purity of CD11b+ CD45+ microglia. Figure S6. Microarray validation with qPCR for lgf-1. Figure S7. The gene expression profile of cortex and SVZ microglia after neonatal HI shared similarities with that of microglia from rodent models of neurodegenerative diseases.
}

\section{Abbreviations}

AD: Alzheimer's disease; ALS: Amyotrophic lateral sclerosis; BRDU: Bromodeoxyuridine; CC: Corpus callosum; CC3: Cleaved caspase 3; CNS: Central nervous system; CX: Cortex; DCX: Doublecortin; DIC: Day in culture; FC: Fold change; FDR: False detection rate; GO: Gene ontology; HBSS: Hank's balanced salt solution; HI: Hypoxia-ischemia; HIE: Hypoxicischemic encephalopathy; Iba1: Ionized calcium-binding adapter molecule 1; KEGG: Kyoto Encyclopedia of Genes and Genomes; LPS: Lipopolysaccharide; MCAO: Middle cerebral artery stroke; NPC: Neural progenitor cell; NSC: Neural stem cell; P: Postnatal day; PAX6: Paired box 6 protein; PB: Phosphate buffer; PCNA: Proliferating nuclear cell antigen; PFA: Paraformaldehyde; PSANCAM: Polysialylated-neural cell adhesion molecule; qPCR: Quantitative realtime polymerase chain reaction; RT: Room temperature; SD: Standard deviation; SOX2: Sex-determining region Y-box 2; SVZ: Subventricular zone; TBS: Tris-buffered saline 


\section{Acknowledgements}

The authors thank Dr. Bernd Schwendele for critical discussions, and Mrs. Pia Bustos, Mrs. Tanja Dittmar, and Dr. Michael Abanto for excellent technical assistance. Calculations were partially performed at sciCORE (http://scicore. unibas.ch/) scientific computing center at the University of Basel.

\section{Authors' contributions}

UF and RG conceived and designed the study. UF, CB, and LC performed research. Data was analyzed by UF and FG and interpreted by UF, CB, and RG. The first manuscript was written by UF with critical revision by $C B$ and RG. All authors read and approved the final manuscript.

\section{Funding}

This work was supported by a Swiss National Science Foundation (SNSF) grant no. 146632, the Department of Surgery of the University Hospital Basel, and the Department of Biomedicine, University of Basel.

\section{Availability of data and materials}

The microarray datasets generated and analyzed during the current study are available in the GEO gene expression omnibus repository (GSE97299). The other datasets used and analyzed during the current study are available from the corresponding author on reasonable request.

\section{Ethics approval and consent to participate}

Animal Ethics License no. 2652, Cantonal veterinary office Basel Stadt, Switzerland

\section{Consent for publication}

The consent for publication was given by all authors.

\section{Competing interests}

The authors declare that they have no competing interests.

\section{Author details}

'Department of Neurology, University Hospital Basel, University Basel, Basel, Switzerland. 'Brain ischemia and regeneration, Department of Biomedicine, University Hospital Basel, University Basel, Basel, Switzerland. 'Bioinformatics Core Facility, Department of Biomedicine, University Basel, Basel, Switzerland. ${ }^{4}$ Swiss Institute of Bioinformatics, Basel, Switzerland. ${ }^{5}$ Department of Neurosurgery, University Hospital Basel, University Basel, Basel, Switzerland. ${ }^{6}$ Faculty of Medicine, University Basel, Basel, Switzerland.

\section{Received: 22 July 2019 Accepted: 8 January 2020}

\section{Published online: 18 January 2020}

\section{References}

1. Günther G, Junker R, Sträter R, Schobess R, Kurnik K, Heller C, et al. Symptomatic ischemic stroke in full-term neonates: role of acquired and genetic prothrombotic risk factors. Stroke. 2000;31:2437-41.

2. Grether JK, Nelson KB. Maternal infection and cerebral palsy in infants of normal birth weight. JAMA. 1997;278:207-11.

3. Vannucci RC, Perlman JM. Interventions for perinatal hypoxic-ischemic encephalopathy. Pediatrics. 1997;100:1004-14.

4. Chicha L, Smith T, Guzman R. Stem cells for brain repair in neonatal hypoxia-ischemia. Child's Nerv Syst. 2014;30:37-46.

5. Nair J, Kumar VHS. Current and emerging therapies in the management of hypoxic ischemic encephalopathy in neonates. Child (Basel, Switzerland) 2018;5:99.

6. Brégère C, Fisch U, Sailer MH, Lieb WS, Chicha L, Goepfert F, et al. Neonatal hypoxia-ischemia in rat increases doublecortin concentration in the cerebrospinal fluid. Eur J Neurosci. 2017;46:1758-67.

7. Iwai M, Ikeda T, Hayashi T, Sato K, Nagata T, Nagano I, et al. Temporal profile of neural stem cell proliferation in the subventricular zone after ischemia/ hypoxia in the neonatal rat brain. Neurol Res. 2006;28:461-8.

8. Yang Z, Levison SW. Perinatal hypoxic/ischemic brain injury induces persistent production of striatal neurons from subventricular zone progenitors. Dev Neurosci. 2007;29:331-40.

9. Sato K. Effects of microglia on neurogenesis. Glia. 2015;n/a-n/a.

10. Thored P, Heldmann U, Gomes-Leal W, Gisler R, Darsalia V, Taneera J, et al. Long-term accumulation of microglia with proneurogenic phenotype concomitant with persistent neurogenesis in adult subventricular zone after stroke. Glia. 2009:57:835-49.

11. Ginhoux F, Greter M, Leboeuf M, Nandi S, See P, Gokhan S, et al. Fate mapping analysis reveals that adult microglia derive from primitive macrophages. Science. 2010;330:841-5.

12. Cunningham $\mathrm{CL}$, Martinez-Cerdeno V, Noctor SC. Microglia regulate the number of neural precursor cells in the developing cerebral cortex. J Neurosci. 2013;33:4216-33.

13. Schafer DP, Lehrman EK, Kautzman AG, Koyama R, Mardinly AR, Yamasaki R, et al. Microglia sculpt postnatal neural circuits in an activity and complement-dependent manner. Neuron. 2012;74:691-705.

14. Squarzoni P, Oller G, Hoeffel G, Pont-Lezica L, Rostaing P, Low D, et al. Microglia modulate wiring of the embryonic forebrain. Cell Rep. 2014:8: 1271-9.

15. Ueno M, Fujita Y, Tanaka T, Nakamura Y, Kikuta J, Ishii M, et al. Layer V cortical neurons require microglial support for survival during postnatal development. Nat Neurosci. Nature Publishing Group. 2013;16:543-51.

16. Shigemoto-Mogami Y, Hoshikawa K, Goldman JE, Sekino Y, Sato K. Microglia enhance neurogenesis and oligodendrogenesis in the early postnatal subventricular zone. J Neurosci. 2014;34:2231-43.

17. Wlodarczyk A, Holtman IR, Krueger M, Yogev N, Bruttger J, Khorooshi R, et al. A novel microglial subset plays a key role in myelinogenesis in developing brain. EMBO J. 2017;36:3292-308.

18. Matcovitch-Natan O, Winter DR, Giladi A, Vargas Aguilar S, Spinrad A, Sarrazin S, et al. Microglia development follows a stepwise program to regulate brain homeostasis. Science. 2016;353:aad8670.

19. Hammond TR, Dufort C, Dissing-Olesen L, Giera S, Young A, Wysoker A, et al. Single-cell RNA sequencing of microglia throughout the mouse lifespan and in the injured brain reveals complex cell-state changes. Immunity. 2019:50:253-271.e6.

20. Bennetta ML, Bennetta C, Liddelowa SA, Ajami B, Zamanian JL, Fernhoff NB, et al. New tools for studying microglia in the mouse and human CNS. Pnas. 2016:E1738-46.

21. Grabert K, Michoel T, Karavolos MH, Clohisey S, Baillie JK, Stevens MP, et al. Microglial brain region-dependent diversity and selective regiona sensitivities to aging. Nat Neurosci. 2016;19:504-16.

22. Hristova M, Cuthill D, Zbarsky V, Acosta-Saltos A, Wallace A, Blight K, et al. Activation and deactivation of periventricular white matter phagocytes during postnatal mouse development. Glia. 2010;58:11-28.

23. Kettenmann $\mathrm{H}$, Hanisch U-K, Noda M, Verkhratsky A. Physiology of microglia. Physiol Rev. 2011;91:461-553.

24. Cuaycong M, Engel M, Weinstein SL, Salmon E, Perlman JM, Sunderam S, et al. A novel approach to the study of hypoxia-ischemia-induced clinical and subclinical seizures in the neonatal rat. Dev Neurosci. 2011:33:241-50.

25. Khazipov R, Zaynutdinova D, Ogievetsky E, Valeeva G, Mitrukhina O, Manent $J-B$, et al. Atlas of the postnatal rat brain in stereotaxic coordinates. Front Neuroanat. 2015:9:161.

26. Freilich RW, Woodbury ME, Ikezu T. Integrated expression profiles of mRNA and miRNA in polarized primary murine microglia. Khoury J El, editor. PLoS One. 2013;8:e79416

27. Ong J, Plane JM, Parent JM, Silverstein FS. Hypoxic-ischemic injury stimulates subventricular zone proliferation and neurogenesis in the neonatal rat. Pediatr Res. 2005:58:600-6.

28. Buono KD, Goodus MT, Guardia Clausi M, Jiang Y, Loporchio D, Levison SW. Mechanisms of mouse neural precursor expansion after neonatal hypoxiaIschemia. J Neurosci. 2015;35:8855-65.

29. Sierra A, Encinas JM, Deudero JJP, Chancey JH, Enikolopov G, OverstreetWadiche LS, et al. Microglia shape adult hippocampal neurogenesis through apoptosis-coupled phagocytosis. Cell Stem Cell. Elsevier Inc. 2010;7:483-95.

30. Fourgeaud L, Través PG, Tufail Y, Leal-Bailey H, Lew ED, Burrola PG, et al. TAM receptors regulate multiple features of microglial physiology. Nature. Nature Publishing Group. 2016:1-15.

31. Rahpeymai Y, Hietala MA, Wilhelmsson U, Fotheringham A, Davies I, Nilsson A-K, et al. Complement: a novel factor in basal and ischemia-induced neurogenesis. EMBO J. 2006;25:1364-74.

32. Holtman IR, Raj DD, Miller JA, Schaafsma W, Yin Z, Brouwer N, et al. Induction of a common microglia gene expression signature by aging and neurodegenerative conditions: a co-expression meta-analysis. Acta Neuropathol Commun. 2015;3:31.

33. Chiu IM, Morimoto ET, Goodarzi H, Liao JT, O'Keeffe S, Phatnani HP, et al. A neurodegeneration-specific gene-expression signature of acutely isolated 
microglia from an amyotrophic lateral sclerosis mouse model. Cell Rep. The Authors; 2013;4:385-401.

34. Chapman KZ, Ge R, Monni E, Tatarishvili J, Ahlenius H, Arvidsson A, et al. Inflammation without neuronal death triggers striatal neurogenesis comparable to stroke. Neurobiol Dis. Elsevier Inc. 2015;83:1-15.

35. Frost JL, Schafer DP. Microglia: architects of the developing nervous system. Trends Cell Biol. Elsevier Ltd. 2016;26:587-97.

36. Parakalan R, Jiang B, Nimmi B, Janani M, Jayapal M, Lu J, et al. Transcriptome analysis of amoeboid and ramified microglia isolated from the corpus callosum of rat brain. BMC Neurosci. 2012;13:64.

37. Solga AC, Pong WW, Walker J, Wylie T, Magrini V, Apicelli AJ, et al. RNAsequencing reveals oligodendrocyte and neuronal transcripts in microglia relevant to central nervous system disease. Glia. 2015;63:531-48.

38. Butovsky O, Ziv Y, Schwartz A, Landa G, Talpalar AE, Pluchino S, et al. Microglia activated by IL-4 or IFN- $\gamma$ differentially induce neurogenesis and oligodendrogenesis from adult stem/progenitor cells. Mol Cell Neurosci. 2006:31:149-60

39. Cacci E, Ajmone-Cat MA, Anelli T, Biagioni S, Minghetti L. In vitro neuronal and glial differentiation from embryonic or adult neural precursor cells are differently affected by chronic or acute activation of microglia. Glia. 2008;56: $412-25$

40. Monje ML, Toda H, Palmer TD. Inflammatory blockade restores adult hippocampal neurogenesis. Science. 2003;302:1760-5.

41. Lalancette-Hébert M, Gowing G, Simard A, Weng YC, Kriz J. Selective ablation of proliferating microglial cells exacerbates ischemic injury in the brain. J Neurosci. 2007;27:2596-605.

42. Szulzewsky F, Pelz A, Feng X, Synowitz M, Markovic D, Langmann T, et al. Glioma-associated microglia/macrophages display an expression profile different from M1 and M2 polarization and highly express Gpnmb and Spp1. Harrison JK, editor. PLoS One. 2015;10:e0116644.

43. Kamphuis W, Kooijman L, Schetters S, Orre M, Hol EM. Transcriptional profiling of CD11c-positive microglia accumulating around amyloid plaques in a mouse model for Alzheimer's disease. Biochim Biophys Acta - Mol Basis Dis. 1862;2016:1847-60.

44. Ripoll VM, Irvine KM, Ravasi T, Sweet MJ, Hume DA. Gpnmb is induced in macrophages by IFN- and lipopolysaccharide and acts as a feedback regulator of proinflammatory responses. J Immunol. 2007;178:6557-66. https://doi.org/10.4049/jimmunol.178.10.6557.

45. Nakano M, Satoh K, Fukumoto Y, Ito Y, Kagaya Y, Ishii N, et al. Important role of erythropoietin receptor to promote VEGF expression and angiogenesis in peripheral ischemia in mice. Circ Res. 2007;100:662-9.

46. Tanaka H, Shimazawa M, Kimura M, Takata M, Tsuruma K, Yamada M, et al. The potential of GPNMB as novel neuroprotective factor in amyotrophic lateral sclerosis. Sci Rep. 2012;2:573.

47. Ransohoff RM. A polarizing question: do M1 and M2 microglia exist? Nat Neurosci. 2016;19:987-91.

48. Colonna M, Butovsky O. Microglia function in the central nervous system during health and neurodegeneration. Annu Rev Immunol. 2017;35:441-68.

49. Srinivasan K, Friedman BA, Larson JL, Lauffer BE, Goldstein LD, Appling LL, et al. Untangling the brain's neuroinflammatory and neurodegenerative transcriptional responses. Nat Commun. 2016;7:11295.

50. Holtman IR, Bsibsi M, Gerritsen WH, Boddeke HWGM, Eggen BJL, van der Valk P, et al. Identification of highly connected hub genes in the protective response program of human macrophages and microglia activated by alpha B-crystallin. Glia. 2017;65:460-73.

51. Walton NM, Sutter BM, Laywell ED, Levkoff LH, Kearns SM, Marshall GP, et al. Microglia instruct subventricular zone neurogenesis. Glia. 2006;54:815-25.

52. Deierborg T, Roybon L, Inacio AR, Pesic J, Brundin P. Brain injury activates microglia that induce neural stem cell proliferation ex vivo and promote differentiation of neurosphere-derived cells into neurons and oligodendrocytes. Neuroscience. Elsevier Inc. 2010;171:1386-96.

53. Liu J, Hjorth E, Zhu M, Calzarossa C, Samuelsson EB, Schultzberg M, et al. Interplay between human microglia and neural stem/progenitor cells in an allogeneic co-culture model. J Cell Mol Med. 2013;17:1434-43.

54. Ortega FJ, Vukovic J, Rodríguez MJ, Bartlett PF. Blockade of microglial KATPchannel abrogates suppression of inflammatory-mediated inhibition of neural precursor cells. Glia. 2014;62:247-58.

55. Covey MV, Loporchio D, Buono KD, Levison SW. Opposite effect of inflammation on subventricular zone versus hippocampal precursors in brain injury. Ann Neurol. 2011;70:616-26.
56. Erickson Rl, Paucar AA, Jackson RL, Visnyei K, Kornblum H. Roles of insulin and transferrin in neural progenitor survival and proliferation. J Neurosci Res. 2008;86:1884-94.

57. Talaverón R, Fernández P, Escamilla R, Pastor AM, Matarredona ER, Sáez JC. Neural progenitor cells isolated from the subventricular zone present hemichannel activity and form functional gap junctions with glial cells. Front Cell Neurosci. 2015;9:411.

58. Mosher Kl, Andres RH, Fukuhara T, Bieri G, Hasegawa-Moriyama M, He Y, et al. Neural progenitor cells regulate microglia functions and activity. Nat Neurosci. Nature Publishing Group. 2012;15:1485-7.

\section{Publisher's Note}

Springer Nature remains neutral with regard to jurisdictional claims in published maps and institutional affiliations.
Ready to submit your research? Choose BMC and benefit from:

- fast, convenient online submission

- thorough peer review by experienced researchers in your field

- rapid publication on acceptance

- support for research data, including large and complex data types

- gold Open Access which fosters wider collaboration and increased citations

- maximum visibility for your research: over $100 \mathrm{M}$ website views per year

At BMC, research is always in progress.

Learn more biomedcentral.com/submissions 